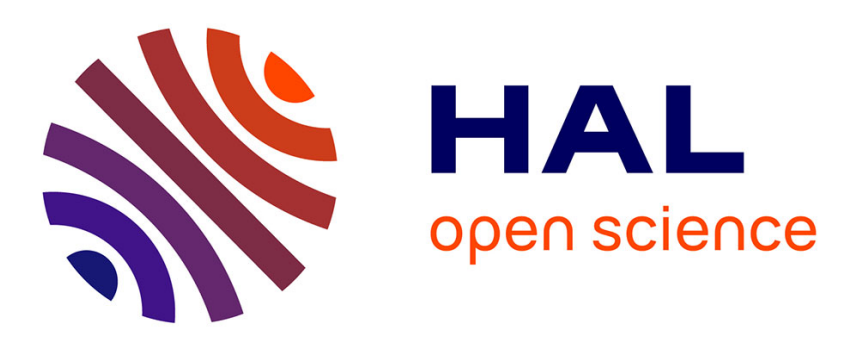

\title{
Relativistic Methods for Calculating Electron Paramagnetic Resonance (EPR) Parameters
}

Hélène Bolvin, Jochen Autschbach

\section{To cite this version:}

Hélène Bolvin, Jochen Autschbach. Relativistic Methods for Calculating Electron Paramagnetic Resonance (EPR) Parameters. Springer. Handbook of Relativistic Quantum Chemistry, Springer Berlin Heidelberg, pp.1-39, 2015, 10.1007/978-3-642-41611-8_12-1 . hal-02483183

\section{HAL Id: hal-02483183 \\ https://hal.science/hal-02483183}

Submitted on 18 Feb 2020

HAL is a multi-disciplinary open access archive for the deposit and dissemination of scientific research documents, whether they are published or not. The documents may come from teaching and research institutions in France or abroad, or from public or private research centers.
L'archive ouverte pluridisciplinaire HAL, est destinée au dépôt et à la diffusion de documents scientifiques de niveau recherche, publiés ou non, émanant des établissements d'enseignement et de recherche français ou étrangers, des laboratoires publics ou privés. 


\title{
Chapter 0 \\ Relativistic methods for calculating Electron Paramagnetic Resonance (EPR) parameters
}

\author{
Hélène Bolvin and Jochen Autschbach
}

\begin{abstract}
Basic concepts for calculating electronic paramagnetic resonance are discussed, with a focus on methods that are suitable for molecules containing heavy elements. Inclusion of relativistic effects is essential in such calculations. Selected examples are presented to illustrate practical applications of these theoretical methods
\end{abstract}

\section{Preliminaries: Units, Notation, Acronyms}

The reader is assumed to be familiar with basic concepts of quantum mechanics - including relativistic methods covered in other chapters - and basic concepts of computational chemistry. SI units are employed. Nuclear motion is not considered; the focus is on electronic structure and the resulting magnetic properties. The symbols $\cdot$ and $\times$ indicate inner and outer products, respectively, for vectors and matrices or tensors. Bold-italic notation such as $\boldsymbol{r}, \hat{\boldsymbol{S}}, \boldsymbol{\mu}$ is used for vectors and vector operators, while upright-bold such as $\mathbf{a}, \mathbf{G}, \boldsymbol{\mu}$ is used for matrices and rank-2 tensors.

The following acronyms are used occasionally in the text:

AO atomic orbital (basis function or actual AO)

CAS complete active space

DFT Density Functional Theory (usually KS, 'pure' and generalized KS variants)

EM electro-magnetic

GIAO gauge-including atomic orbital

HF Hartree-Fock

HFC hyperfine coupling

Please address correspondence to either one of the authors. Hélène Bolvin, Laboratoire de Physique et de Chimie Quantiques, Université Toulouse 3, 118 Route de Narbonne, 31062 Toulouse, France, e-mail: bolvineirsamc.ups-tlse. fr. Jochen Autschbach, Department of Chemistry, University at Buffalo, State University of New York, e-mail: jochena@buffalo. edu 


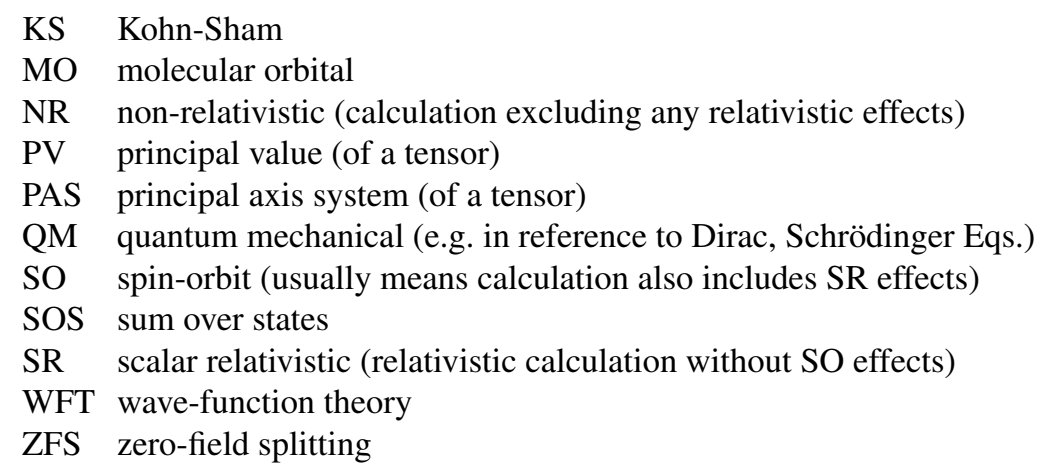

\subsection{Introduction and Background: EPR parameters and Spin Hamiltonians}

Many of the chemical species encountered in the laboratory and in everyday life have non-degenerate closed shell ground states. But there are also many exceptions, such as open shell metal complexes, stable radicals, and most atoms. In the absence of external electromagnetic (EM) fields, such species may afford a degenerate electronic ground state and degenerate excited states. Species with closed-shell ground states may also afford degenerate excited states such as excited spin triplets. The term degeneracy means that an electronic state $\lambda$ with energy $E_{\lambda}$ may have $d_{\lambda}$ state components $|\lambda, a\rangle$, with $a=1 \ldots d_{\lambda}$, such that each $|\lambda, a\rangle$ and any linear combination thereof is a solution to the field-free quantum mechanical $(\mathrm{QM})$ equation describing the system (e.g. Schrödinger equation, Dirac equation, approximate twocomponent relativistic QM methods, as discussed elsewhere in this Handbook) with the same energy $E_{\lambda}$. The index $\lambda$ may simply be a number counting the energy levels of the system, or it may be a spectroscopic symbol characterizing a state of interest, or a symmetry label, or a combination thereof. The discussion excludes cases of accidental degeneracy.

Electron paramagnetic resonance (EPR) [1-4] is a primary tool for studying degenerate and nearly degenerate electronic states experimentally. An external magnetic field $\boldsymbol{B}$ splits the degeneracy (Zeeman effect) to yield a new set of states. EM radiation of a suitable frequency may then induce transitions among them and allow to measure the energy splittings spectroscopically. The parameters extracted from the spectra (vide infra) contain a wealth of information about the electronic structure and molecular structure.

To illustrate the effect utilized in EPR spectroscopy, consider a single unpaired electron and - first - neglect spin-orbit (SO) coupling. This situation represents a spin doublet (a two-fold degenerate state) with spin quantum number $S=1 / 2$. If there is no external magnetic field, the two possible orientations of the spin projection onto a quantization axis $\left(M_{S}= \pm 1 / 2\right)$ have the same energy. Associated with the spin angular momentum vector $\boldsymbol{S}$ is a magnetic dipole moment $\boldsymbol{\mu}_{e}=-g_{e} \beta_{e} \boldsymbol{S}$, 
with $\beta_{e}=e \hbar /\left(2 m_{e}\right)$ being the Bohr magneton and $g_{e}$ the free electron $g$-value or $g$ factor with a current experimental value [5] of 2.00231930436153 (53). The Dirac equation predicts $g_{e}=2$ exactly. Therefore, $g_{e}=2$ is used occasionally in the following. The small differences in the values of $g_{e}$ are due to quantum electrodynamic corrections.

For $S=1 / 2$ the magnetic moment associated with the spin has two projections onto a quantization axis. If a static external magnetic field is applied, the direction of $\boldsymbol{B}$ defines the quantization axis. With the field present, the quantized projections of $\boldsymbol{\mu}_{e}$ do not have the same energy. In classical physics, the energy of a magnetic dipole $\boldsymbol{\mu}$ in a magnetic field $\boldsymbol{B}$ is

$$
E=-\boldsymbol{\mu} \cdot \boldsymbol{B}
$$

The lowest energy is for $\boldsymbol{\mu}$ and $\boldsymbol{B}$ being anti-parallel, and the highest energy is for $\boldsymbol{\mu}$ and $\boldsymbol{B}$ being parallel. This is the physical mechanism that keeps a compass needle pointing toward the magnetic north pole. Quantum mechanically, a semi-classical Zeeman Hamiltonian that describes such an effect for a quantized spin magnetic moment is

$$
\hat{\mathcal{H}}^{\mathrm{Z}}=g_{e} \beta_{e} \hat{\boldsymbol{S}} \cdot \boldsymbol{B}
$$

with $\hat{\boldsymbol{S}}$ being the spin vector operator. The negative sign in $(0.1)$ is canceled by the negative sign relating $\boldsymbol{\mu}_{e}$ to the spin. One may choose a coordinate system such that $\boldsymbol{B}$ is oriented along the $z$ axis, with amplitude $B_{0}$. The Hamiltonian (0.2) then reads

$$
\hat{\mathcal{H}}^{\mathrm{Z}}=g_{e} \beta_{e} B_{0} \hat{S}_{z}
$$

The eigenvalues are those of $\hat{S}_{z}$ times $g_{e} \beta_{e} B_{0}$, i.e. $\pm(1 / 2) g_{e} \beta_{e} B_{0}$. The magnetic field lifts the degeneracy of the spin doublet. A transition from the lower energy level ( $\boldsymbol{B}$ and $\boldsymbol{S}$ 'anti-parallel') to the higher one ( $\boldsymbol{B}$ and $\boldsymbol{S}$ 'parallel') requires an energy of

$$
\Delta E=h \nu=g_{e} \beta_{e} B_{0}
$$

In the equation above, $\nu$ is the frequency of EM radiation used to induce the transition. The frequency is approximately $28 \mathrm{GHz} / \mathrm{T}$. In a typical EPR spectrometer, $B_{0}$ is $0.34 \mathrm{~T}$ ( 3,400 Gauss), which translates to a free electron spin-1/2 resonance frequency of about $9.5 \mathrm{GHz}$. This is radiation in the X-band microwave region of the EM spectrum. For general spin values $S$, the degeneracy of the projection is $2 S+1$. A magnetic field splits these into $2 S+1$ individual states. Absorption of a photon of EM radiation entails conservation of angular momentum. The photon has an angular momentum of $\hbar$, corresponding to one atomic unit (au), and therefore the selection rule is that transitions with $\Delta M_{S}=1$ are allowed.

One may repeat the calculation for the spin $\boldsymbol{I}_{K}$ of some nucleus no. $K$, by substituting the electron spin magnetic dipole moment by the nuclear spin magnetic dipole moment $\boldsymbol{\mu}_{K}=g_{K} \beta_{N} \boldsymbol{I}_{K}$. Here, $g_{K}$ is the $g$-factor for a given nuclear isotope, and $\beta_{N}=e \hbar /\left(2 M_{P}\right)$ is the nuclear magneton, with $M_{P}$ being the proton mass. The latter is approximately 2000 times greater than the electron mass, and therefore the associated transition energies for nuclear spins are in the radio-frequency range 
(tens of $\mathrm{MHz}$ ) at magnetic field strengths used in EPR spectrometers. Transitions between nuclear spin projections are observed directly in nuclear magnetic resonance (NMR). At EPR spectrometer field strengths, the energy splitting for nuclear transitions is very small and one may assume equal Boltzmann populations of the nuclear spin projection states in the absence of other magnetic fields. However, there is a magnetic coupling of electronic and nuclear magnetic moments called hyperfine coupling (HFC). Hyperfine coupling gives rise to hyperfine structure of EPR spectra when nuclei with non-zero spin are present.

There is another type of fine structure that can be observed in EPR experiments for systems with $S \geq 1$. For a given magnetic field strength $B_{0}$, the $2 S+1$ spin projections with different $M_{S}$ would be expected to be equally spaced energetically, meaning that any of the possible $2 S$ allowed transitions with $\Delta M_{S}=1$ give resonances at the same frequency. However, at lower field strengths the spectra may exhibit $2 S$ distinct features at lower field strengths [1], which indicates unequal energetic separations of the $M_{S}$ components in the presence of the field. This fine structure can be traced back to a removal of the degeneracy of the spin multiplet already at zero field. The effect is therefore called zero field splitting (ZFS). A spin $S \geq 1$ implies that there are two or more unpaired electrons. The physical origin of ZFS is a magnetic interaction between pairs of electrons, either directly (a dipolar spin-spin interaction arising from relativistic corrections to the electron-electron interaction) or mediated via SO coupling (which has one- and two-electron contributions). The ZFS can therefore be associated with relativistic effects. For systems with heavy elements, as well as for many lighter systems, the SO contribution to ZFS is the dominant one.

The observed electronic magnetic moment resulting from an electronically degenerate state may differ from what is expected based solely on the spin quantum number. For instance there may be an orbital degeneracy present in an electronic state, meaning that the observed magnetic moment is not only due to an electron spin but also due to an orbital angular momentum. The interaction with the external field can be expressed via the total angular momentum $\boldsymbol{J}$, which is obtained from the vector addition of the quantized spin and orbital angular momenta, $\boldsymbol{S}$ and $\boldsymbol{L}$. As an example, the $S=1 / 2, L=1\left({ }^{2} P\right)$ ground state of the fluorine atom has a total angular momentum quantum number $J=3 / 2$, with total spin and orbital angular momentum parallel, giving a 4-fold degenerate state $\left(M_{J}\right.$ ranging from $+3 / 2$ to $-3 / 2$ ) whose components split in the presence of a magnetic field. Transitions in the EPR experiment may be observed for $\Delta M_{J}=1$. Instead of Equation (0.4), the transition frequencies are determined by

$$
\Delta E=h \nu=g_{J} \beta_{e} B_{0}
$$

with $g_{J}=4 / 3$ for the fluorine ${ }^{2} P_{3 / 2}$ state, instead of 2. Here, $g_{J}$ is the Landé $g$ factor; the experimentally observed $g$-factor obtained from matching the measured resonance frequency with Equation (0.5) for known field strength $B_{0}$ is very close to this number. The large difference of $g_{J}$ from the free-electron $g_{e}=2$ arises because the state reflects not only a spin doublet but also an orbital triplet. 
In the absence of orbital degeneracy, an orbital magnetic moment and deviations of observed $g$-factors from the free electron value may arise because of SO coupling. For organic doublet radicals with only light elements, $S=1 / 2, M_{S}= \pm 1 / 2$ are basically good quantum numbers. However, even in this situation the observed $g$ factors may differ from 2 (typically ranging from 1.9 to 2.1) because of SO coupling. Because of the small deviations, it is sometimes preferred to report $g$-shifts $\Delta g=$ $g-g_{e}$ (often in units of parts per thousand (ppt)), in analogy to NMR chemical shifts. Since SO coupling is a relativistic effect, the presence of $g$-shifts for orbitally nondegenerate states directly indicates relativistic effects. If SO coupling is strong, $S$ and $M_{S}$ may not be good quantum numbers at all. In this case, $g$-shifts can become very large even in the absence of orbital degeneracy (meaning an absence of orbital angular momentum in the corresponding scalar relativistic (SR) state). A case in point is the doublet ground state of $\mathrm{NpF}_{6}$ for which the observed [6] $g$-factor is -0.6 .

To summarize: A degenerate paramagnetic electronic state gives rise to a more or less complicated pattern of EPR resonances. Given the potential influence of SO coupling, orbital angular momenta, and ZFS, the spectrum is usually interpreted and quantified by invoking the concept of a pseudo-spin $\mathscr{S}$ rather than the actual electron spin $S$. The value of $\mathscr{S}$ defines the degeneracy, $2 \mathscr{S}+1$, of the state that is split by the magnetic field into components with different $\mathscr{M}_{\mathscr{S}}$. The various effects discussed above can be included in a phenomenological pseudo-spin Hamiltonian, which, in lowest order, reads

$$
\hat{\mathcal{H}}_{S}=\beta_{e} \boldsymbol{B} \cdot \mathbf{g} \cdot \hat{\mathscr{S}}+\boldsymbol{I}_{K} \cdot \mathbf{a}_{K} \cdot \hat{\mathscr{S}}+\hat{\mathscr{S}} \cdot \mathbf{d} \cdot \hat{\mathscr{S}}
$$

The parameters in the Hamiltonian are determined by requiring that the transitions with $\Delta \mathscr{M}_{\mathscr{S}}=1$ between its eigenstates reproduce the observed spectrum. The spin Hamiltonian is designed such that its elements within the set of fictitious spin eigenstates are the same as the matrix elements of the true Hamiltonian within the set of true eigenstates. It supposes a correspondence between pseudo-spin and true eigenstates, up to a phase factor common to all the vectors. While this assignment can be rather arbitrary, the basic requirement is that the spin Hamiltonian in the fictitious space transforms in coherence as the real Hamiltonian does in the real space, either by time inversion or by the spatial symmetries of the molecule. [7]

On the right-hand side of Equation (0.6) are, from left to right, the pseudo-spin operators for the Zeeman interaction, the HFC interaction, and the ZFS. Only the pseudo-spin related to the electronic state is treated quantum mechanically. The nuclear spin and external magnetic field are parameters. In Equation (0.6), $\mathbf{g}, \mathbf{a}_{K}$, and $\mathbf{d}$ are $3 \times 3$ matrices parametrizing the various interactions. The fact that they are written in matrix form reflects the possibility that the observed interactions may be anisotropic. For example, observed $g$-factors for a molecule with axial symmetry may be very different if the magnetic field is oriented along the axial direction or perpendicular to it. Higher order terms requiring additional sets of parameters in Equation (0.6) may be required to reflect the full complexity of an EPR spectrum, as discussed in Section 0.3. 
The matrices $\mathbf{g}$ and $\mathbf{a}_{K}$ are often referred to as the $g$-'tensor' and the HFC 'tensor'. It was pointed out in the book by Abragam \& Bleaney [4] that they are in fact not proper rank-2 tensors. Following a suggestion by Atherton [1], one may refer to them as the Zeeman coupling matrix $(\mathbf{g})$ and the HFC matrix $\left(\mathbf{a}_{K}\right)$ instead. The $g$-factor observed for a magnetic field in the direction of a unit vector $\boldsymbol{u}$ in the laboratory coordinate system is given by

$$
g_{\boldsymbol{u}}= \pm\left(\boldsymbol{u} \cdot \mathbf{g g}^{T} \cdot \boldsymbol{u}\right)^{1 / 2}
$$

(superscript $T$ indicating a matrix transpose). One may define a hyperfine coupling associated with a particular quantization direction chosen for the pseudo-spin in a similar way. The corresponding objects $\mathbf{G}=\mathbf{g g}^{T}$ and $\mathbf{A}_{K}=\mathbf{a}_{K} \mathbf{a}_{K}^{T}$ are rank-2 tensors whose eigenvalues and eigenvectors define the squares of the principal values (PVs) of $\mathbf{g}$ and $\mathbf{a}_{K}$ and a principal axis system (PAS) of each of the interactions. For example, the principal $g$-factors correspond to the $g$-factors that are observed when the direction of the magnetic field $\boldsymbol{B}$ coincides with one of the principal axes. Section 0.4 addresses the question of the signs of the PVs of the Zeeman and HFC interaction in more detail.

Section 0.2 sketches different computational relativistic methods by which to obtain the EPR parameters in the spin Hamiltonian of Equation (0.6) from first principles. As already mentioned, SO coupling plays an important role. HFC can also be strongly impacted by SR and SO relativistic effects. Selected illustrative examples are presented in Section 0.5.

\subsection{Computational methods for EPR parameter calculations}

\subsubsection{Representation of the pseudo-spin Hamiltonian in an ab-initio framework}

Equation (0.6) and generalizations thereof presents some conceptual challenges when addressing the problem by relativistic or non-relativistic (NR) molecular quantum mechanics. The reason is that the pseudo-spin operator $\hat{\mathscr{S}}$ may have little in common with the electron spin operator $\hat{\boldsymbol{S}}$ if SO coupling or ZFS are strong, or if the orbital angular momenta are not quenched. What can be done instead follows roughly the following sequence [3], if a calculation starts out with a pure spinmultiplet (i.e. from a NR or SR reference without orbital degeneracy) and if effects from SO coupling can be dealt with as a perturbation:

- Define a Hamiltonian $\hat{\mathcal{H}}_{0}$ for the system in the absence of external EM fields and find its eigenstates with energies $E_{\lambda}$, spin degeneracies $d_{\lambda}$, and corresponding orthonormal QM eigenfunctions $|\lambda, a\rangle$.

- Consider a perturbation: a homogeneous external field $\boldsymbol{B}$ for the Zeeman interaction, the hyperfine magnetic field from a nuclear spin magnetic moment $\boldsymbol{\mu}_{K}$, or 
spin-dependent perturbations. Define a corresponding perturbation Hamiltonian $\hat{\mathcal{H}}^{\prime}$. The effects from SO coupling may also be absorbed into $\hat{\mathcal{H}}^{\prime}$. The perturbations are assumed to be weak enough such that one can identify the eigenstates of $\hat{\mathcal{H}}_{0}+\hat{\mathcal{H}}^{\prime}$ corresponding to a multiplet $\lambda$ of $\hat{\mathcal{H}}_{0}$ of interest. Diagonalization of the matrix $\mathbf{H}_{0}+\mathbf{H}^{\prime}$ representing the Hamiltonian in the complete set (or a large subset) of eigenstates of $\hat{\mathcal{H}}_{0}$, or finding a selected number of eigenfunctions and eigenvalues with techniques such as the Davidson or Lanczos algorithms, would give the eigenfunctions and energies for the perturbed system. Typically, there would be a mixing among eigenvectors belonging to a multiplet $\lambda$ as well as some admixture from components of other multiplets.

- Instead, select a multiplet $\lambda$ with degeneracy $d_{\lambda}$ of interest corresponding to $\hat{\mathcal{H}}_{0}$, and seek a $d_{\lambda} \times d_{\lambda}$ matrix representation $\mathbf{H}_{\text {eff }}$ of an effective Hamiltonian $\hat{\mathcal{H}}_{\text {eff }}$ with the following properties: (i) the eigenvalues of $\mathbf{H}_{\text {eff }}$ are the same as those of $\mathbf{H}_{0}+\mathbf{H}^{\prime}$ for the perturbed multiplet $\lambda$. (ii) The eigenvectors of $\mathbf{H}_{\text {eff }}$ describe how the components of the unperturbed multiplet mix under the perturbation. There are various ways by which $\mathbf{H}_{\text {eff }}$ can be calculated. A well-known approach is by perturbation theory as an approximation to second order, which gives for a matrix element related to the multiplet $\lambda$ :

$$
\left\langle\lambda a\left|\hat{\mathcal{H}}_{\mathrm{eff}}\right| \lambda a^{\prime}\right\rangle=\delta_{a a^{\prime}} E_{\lambda}+\left\langle\lambda a\left|\hat{\mathcal{H}}^{(a)}\right| \lambda a^{\prime}\right\rangle-\sum_{\mu \neq \lambda} \sum_{b=1}^{d_{\mu}} \frac{\left\langle\lambda a\left|\hat{\mathcal{H}}^{(b)}\right| \mu b\right\rangle\left\langle\mu b\left|\hat{\mathcal{H}}^{(c)}\right| \lambda a\right\rangle}{E_{\lambda}-E_{\mu}}
$$

Here, $\hat{\mathcal{H}}^{(a)}, \hat{\mathcal{H}}^{(b)}$, and $\hat{\mathcal{H}}^{(b)}$ are parts of $\hat{\mathcal{H}}^{\prime}$ such that the overall matrix element (minus the $\delta_{a a^{\prime}} E_{\lambda}$ part) affords terms that are linear in $\boldsymbol{B}$ or $\boldsymbol{I}_{K}$, or bi-linear in electron spin operators.

- Apart from a constant shift on the diagonal, the matrix representation of the pseudo-spin Hamiltonian, Equation (0.6), written in terms of $\left|\mathscr{M}_{\mathscr{S}}\right\rangle$ pseudo-spin projects is then supposed to have the same elements as those of $\mathbf{H}_{\text {eff }}$. For the Zeeman interaction, the contribution to $\mathbf{H}_{\text {eff }}$ should be linear in $\boldsymbol{B}$. Then, $\hat{\mathcal{H}}^{(a)}$ and $\hat{\mathcal{H}}^{(b)}$ are the Zeeman operator (in a suitable relativistic form) and $\hat{\mathcal{H}}^{(c)}$ additionally considers SO effects. If $\hat{\mathcal{H}}^{(a)}$ and $\hat{\mathcal{H}}^{(b)}$ are instead QM operators linear in $\boldsymbol{I}_{K}$ describing the nuclear hyperfine field, a mapping onto the HFC part of the pseudo-spin Hamiltonian can be made. Finally, if $\hat{\mathcal{H}}^{(a)}$ is the dipolar spin-spin interaction operator, and $\hat{\mathcal{H}}^{(b)}$ and $\hat{\mathcal{H}}^{(c)}$ represent SO interactions, one obtains an effective Hamiltonian quadratic in the electron spin which represents ZFS.

In the previous approach, the matching between real and fictitious states is made according to $|\lambda, M\rangle \equiv\left|\mathscr{S}, \mathscr{M}_{\mathscr{S}}\right\rangle$ since the model space is a spin multiplet; it supposes a similarity between the real spin and the pseudo-spin. This is only valid in the weak SO limit but the procedure permits the calculation of the spin Hamiltonian parameters for all values of the pseudo-spin $\mathscr{S}$ [8]. Equation (0.8) misses quadratic contributions which may be important [9].

The value of the pseudo-spin $\mathscr{S}$ defines the size of the model space. If the state of interest, usually the ground state, is $d$-fold degenerate or nearly degenerate, $\mathscr{S}$ is chosen such that $d=2 \mathscr{S}+1$. In the case of weak SO coupling and a spatially 
non degenerate state ${ }^{2 S+1} \Gamma$ where $\Gamma$ is a non degenerate irreducible representation (irrep) of the system's point group, the SO coupling with the excited states splits the spin degeneracy. This ZFS splitting is on the order of a few $\mathrm{cm}^{-1}$ and gives the fine structure to the EPR spectra. In some cases, the ZFS splitting may be on the order of several ten $\mathrm{cm}^{-1}$ and high-field high-frequency EPR (HF-HF EPR) is necessary to detect the transitions between these components. In this case, one usually takes $\mathscr{S}=S$. When the ground state is spatially degenerate or if there are very low lying excited states, there are large orbital contributions to the magnetic moment and the choice of the spin Hamiltonian becomes more complicated and must be treated case by case.

For systems where $\mathrm{SO}$ coupling is strong, a close correspondence of a degenerate electronic state of interest with an electron spin multiplet may simply not exist. A corresponding QM method used to determine the electronic states may already include SO coupling in some form, possibly along with a spin-spin interaction term. In this case, the ZFS effects are included in the electronic spectrum. It is shown next how the pseudo-spin Hamiltonian parameters for the Zeeman and HFC interactions can be extracted from such QM calculations. For further discussion see Section 0.3.

When the SO coupling is large, the degeneracy of the states is related to symmetry. For odd-electron systems, the degeneracy is even due to Kramers' theorem. Four-fold degenerate irreps only appear in the cubic and icosahedral groups, along with six-fold in the latter. Therefore, except for highly symmetric molecules, the ground-state of Kramers systems is modeled using $\mathscr{S}=1 / 2$. For even-electron systems, if there is a high-order rotation axis, states may be doubly degenerate and $\mathscr{S}=1 / 2$ represents a non-Kramers doublet. Only cubic and icosahedral groups may have higher degeneracies. Therefore, the states of even-electron systems with a heavy elements are usually non-degenerate or in the case of symmetry, can be considered as non-Kramers doublets. In lanthanide and actinide complexes, the term of the free ion ${ }^{2 S+1} L_{J}$ is split due to the environment of the ligands. This splitting is usually on the order of some tens of $\mathrm{cm}^{-1}$ for lanthanides since the $4 f$ orbitals are mostly inner-shell and interact weakly with the environment. The splitting of the free ion term of an actinide is larger since the $5 f$ orbitals interact more with the ligands, even forming covalent bonds for the early actinides; it can be on the order of several hundred $\mathrm{cm}^{-1}$. Therefore, in the case of heavy elements, states are at the most doubly degenerate or nearly degenerate and there are usually no EPR transitions with excited states, except for cubic systems.

An ab-initio calculation provides the $2 \mathscr{S}+1$ quasi-degenerate wave functions $|\lambda, a\rangle_{a=1,2 \mathscr{S}+1}$ in the absence of external magnetic field, defining the model space, and the corresponding energies $E_{a}$. Let

$$
\hat{\mathcal{H}}^{Z}=-\beta_{e} \hat{\boldsymbol{\mu}} \cdot \boldsymbol{B}
$$

be the Zeeman operator, with $\hat{\boldsymbol{\mu}}$ being a corresponding dimensionless time-odd QM magnetic moment operator. The Zeeman interaction is characterized by the three matrices of the magnetization operator $\left(\boldsymbol{\mu}^{u}\right)_{a, b}=\left\langle\lambda, a\left|\hat{\mu}_{u}\right| \lambda, b\right\rangle$ with $a, b \in$ $[1,2 \mathscr{S}+1]$ and $u=x, y, z$ being defined in the physical space. Hyperfine matrices 
can be defined analogously. These matrices are further discussed in Sections 0.3 and 0.4 .

The approach is outlined in this section for the Zeeman interaction and a doublet of Kramers states $(\mathscr{S}=1 / 2)$. In this case, there is no ZFS and the spin Hamiltonian reduces to the Zeeman term. In the basis of the pseudo-spin projection eigenfunctions $\left|\mathscr{M}_{\mathscr{S}}\right\rangle$, the operator $\hat{\mathscr{S}}_{k}(k=x, y, z)$ is represented by a matrix $\mathbf{S}_{k}=(1 / 2) \boldsymbol{\sigma}_{k}$, with $\boldsymbol{\sigma}_{k}$ being one of the $2 \times 2$ Pauli spin matrices. The magnetic field vector is expressed in terms of its components as $\boldsymbol{B}=\left(B_{1}, B_{2}, B_{3}\right)$. The matrix representation of the spin Hamiltonian for the Zeeman interaction then reads

$$
\mathbf{H}=\sum_{k} h_{k} \mathbf{S}_{k} \quad \text { with } \quad h_{k}=\beta_{e} \sum_{l} B_{l} g_{l k}
$$

The eigenvalues are easily obtained as \pm the square roots of the eigenvalues of $\mathbf{H}^{2}$, which is already diagonal because of $\mathbf{S}_{k} \mathbf{S}_{l}+\mathbf{S}_{l} \mathbf{S}_{k}=\left(\delta_{k l} / 2\right)\left(\begin{array}{ll}1 & 0 \\ 0 & 1\end{array}\right)$ :

$$
\mathbf{H}^{2}=\frac{1}{4}\left(\begin{array}{cc}
\sum_{k} h_{k}^{2} & 0 \\
0 & \sum_{k} h_{k}^{2}
\end{array}\right)
$$

Therefore, the energy difference $\Delta E$ for the two spin projections is $2\left[(1 / 4) \sum_{k} h_{k}^{2}\right]^{1 / 2}=\left[\sum_{k} h_{k}^{2}\right]^{1 / 2}$, i.e.

$$
\Delta E=\beta_{e}\left[\sum_{k, l} B_{k} B_{l} \sum_{m} g_{k m} g_{l m}\right]^{1 / 2}=\beta_{e}\left[\sum_{k, l} B_{k} B_{l} G_{k l}\right]^{1 / 2}
$$

In the previous equation, $G_{k l}$ is an element of the tensor $\mathbf{G}$ introduced below Equation (0.7).

Next, consider a quantum mechanical framework with a doublet state with two wavefunction components, $\psi_{1}, \psi_{2}$, assumed to be orthonormal for convenience. Further, for the time being it is assumed that the doublet components $\psi_{1}$ and $\psi_{2}$ have the time reversal properties of a Kramers pair. In the basis $\left\{\psi_{1}, \psi_{2}\right\}$, the QM Zeeman operator can also be expressed with the help of the spin-1/2 matrices, as

$$
\mathbf{H}^{\prime}=\sum_{k} h_{k}^{\prime} \mathbf{S}_{k} \quad \text { with } \quad \begin{aligned}
h_{1}^{\prime} & =-2 \beta_{e} \operatorname{Re}\left\langle\psi_{2}|\hat{\boldsymbol{\mu}}| \psi_{1}\right\rangle \cdot \boldsymbol{B} \\
h_{2}^{\prime} & =-2 \beta_{e} \operatorname{Im}\left\langle\psi_{2}|\hat{\boldsymbol{\mu}}| \psi_{1}\right\rangle \cdot \boldsymbol{B} \\
h_{3}^{\prime} & =-2 \beta_{e}\left\langle\psi_{1}|\hat{\boldsymbol{\mu}}| \psi_{1}\right\rangle \cdot \boldsymbol{B}
\end{aligned}
$$

Note that $\left\langle\psi_{1}|\hat{\boldsymbol{\mu}}| \psi_{1}\right\rangle \cdot \boldsymbol{B}=-\left\langle\psi_{2}|\hat{\boldsymbol{\mu}}| \psi_{2}\right\rangle \cdot \boldsymbol{B}$ because of the time reversal symmetry. As with the pseudo-spin Hamiltonian, one can calculate twice the square root of the eigenvalues of $\mathbf{H}^{\prime 2}$ to obtain the energy splitting in the presence of a magnetic field. The result can be rearranged as follows:

$$
\Delta E=\left[\sum_{k}{h_{k}^{\prime}}^{2}\right]^{1 / 2}=\beta_{e}\left[2 \sum_{k, l} B_{k} B_{l} \sum_{a=1}^{2} \sum_{b=1}^{2}\left\langle\psi_{a}\left|\hat{\mu}_{k}\right| \psi_{b}\right\rangle\left\langle\psi_{b}\left|\hat{\mu}_{l}\right| \psi_{a}\right\rangle\right]^{1 / 2}
$$


A factor $1 / 2$ enters inside the square root when the double sum is introduced, to avoid double counting of contributions. By comparison of Equations (0.14) and (0.12), one finds for the elements of the tensor $\mathbf{G}$ :

$$
G_{k l}=2 \sum_{a=1}^{2} \sum_{b=1}^{2}\left\langle\psi_{a}\left|\hat{\mu}_{k}\right| \psi_{b}\right\rangle\left\langle\psi_{b}\left|\hat{\mu}_{l}\right| \psi_{a}\right\rangle
$$

At this point, the assumption that $\psi_{1}$ and $\psi_{2}$ transform as a Kramers pair can be dropped, because any linear combination obtained from $\left\{\psi_{1}, \psi_{2}\right\}$ by unitary transformation gives the same tensor $\mathbf{G}$ from Equation (0.15). Therefore, a computation of $\mathbf{G}$ can utilize a pair of doublet wavefunction components without imposing time reversal symmetry explicitly. The reader is reminded that the definition of the magnetic moment operator components in Equation (0.15) excludes pre-factors of $\beta_{e}$. As written, Equation (0.15) assumes a complete one-particle basis set to represent $\psi_{1}$ and $\psi_{2}$, such that there is no dependence of the results on the gauge origin chosen for the external magnetic field. In calculations with finite basis sets, an origin dependence can be avoided by adopting a distributed gauge origin such a gauge-including atomic orbitals (GIAOs). When distributed origin methods are not available, calculations of magnetic properties of complexes with one paramagnetic metal center often place the metal center at the gauge origin.

The eigenvectors of $\mathbf{G}$ represent the molecule-fixed PAS of the Zeeman interaction, sometimes referred to as the 'magnetic axes' of the system under consideration [10]. The square roots of the eigenvalues are absolute values of the principal $g$-factors. The signs of the $g$-factors are not obtained directly. For further discussion, see Section 0.4.

The tensor A plays an analogous role for HFC as G plays for the Zeeman interaction. Therefore, after a QM operator $\hat{\boldsymbol{F}}_{K}$ has been defined for the hyperfine interaction as follows:

$$
\hat{\mathcal{H}}^{\mathrm{HFC}}=\hat{\boldsymbol{F}}_{K} \cdot \boldsymbol{\mu}_{K}=g_{K} \beta_{N} \hat{\boldsymbol{F}}_{K} \cdot \boldsymbol{I}_{K}
$$

the HFC tensor for a Kramers doublet can be calculated via

$$
A_{k l}=2\left(g_{K} \beta_{N}\right)^{2} \sum_{a=1}^{2} \sum_{b=1}^{2}\left\langle\psi_{a}\left|\hat{F}_{K k}\right| \psi_{b}\right\rangle\left\langle\psi_{b}\left|\hat{F}_{K l}\right| \psi_{a}\right\rangle
$$

For hyperfine coupling, a natural choice for the gauge origin of the hyperfine field is the nucleus for which the HFC tensor is calculated.

A different route has been proposed [11] for calculating $\mathbf{G}$ for arbitrary values of $\mathscr{S}$, which is briefly discussed in Section 0.3 . The expression given in Equation $(0.45)$ is the same as $(0.15)$ for $\mathscr{S}=1 / 2$. As for the $\mathscr{S}=1 / 2$ case, the expression for $\mathbf{G}$ of Equation (0.45) should be adaptable for calculations of HFC. 


\subsubsection{Wavefunction based methods for EPR calculations}

Popular starting points for wavefunction-based computations of EPR parameters are complete active space self consistent field (CASSCF) calculations and related restricted and generalized active space approaches [12], often followed by perturbation-theory (PT) based treatments of dynamic correlation on top of CASSCF. For the latter, second-order perturbation theory (CASPT2) [13] and $n$ electron valence state perturbation theory (NEVPT) [14] are in relatively widespread use. Limitations arise from an insufficient description of spin polarization with the size of active spaces commonly achievable in these types of calculations, which is detrimental for HFC calculations. $g$-factors, ZFS parameters, and magnetic susceptibilities, on the other hand, can be obtained with good accuracy. Recently developed combinations of active-space methods with density matrix renormalization group (DMRG) techniques allow for larger active spaces, which is beneficial for treating electron correlation as well as spin polarization. 'Proof of concept' calculations of HFC appear promising [15]. Linear response methods have also been developed for multi-configurational SCF wavefunctions in order to generate spin polarization suitable for HFC calculations without the need of very large active spaces [16]. In principle, multi-reference coupled-cluster (MRCC) methods should be suitable for EPR parameter calculations. To the authors' knowledge, relativistic MRCC calculations have not been used to predict EPR parameters at the time of writing this article.

Relativistic effects have been / can be included in wavefunction-based EPR calculations in a variety of ways, for instance: (i) by using all-electron SR Hamiltonians or SR effective core potentials (ECPs) to generate wavefunctions for a range of electronic states, followed by treatment of SO coupling via state-interaction (SI), [17] (ii) by including SR and SO effects either via an all-electron Hamiltonian [18] or with ECPs from the outset, (iii) by calculating SR components of a spin-multiplet and treatment of SO coupling as a perturbation in the EPR step [3]. In case (ii), SO effects are treated fully variationally whereas in case (i) a SO Hamiltonian matrix is calculated in a limited basis of active-space wavefunctions and subsequently diagonalized. Approach (iii) is applicable in the weak SO coupling limit. Note that without application of specialized techniques the use of a relativistic ECP for a given atom prevents calculations of the HFC for the same atom because the inner core nodal structure of the valence orbitals is needed. An order-by-order treatment of SO coupling via perturbation theory is also viable, for instance based on fourcomponent relativistic perturbation theory [19] after separation of SR and SO components of the QM operators.

\subsubsection{Hartree-Fock and Kohn-Sham methods for EPR calculations}

The approaches to obtaining EPR parameters outlined above assume that the wavefunction components of a degenerate state of interest are available explicitly from 
a calculation. With single-reference methods such as Hartree-Fock (HF) theory and Kohn-Sham (KS) Density Functional Theory (DFT) and generalized KS methods, the usual approach in the absence of strong SO coupling is to start from a spinunrestricted SR calculation. For brevity, HF theory is considered as a special case of a generalized KS hybrid functional from here on. The use of a spin-unrestricted single-determinant reference typically leads to spin contamination: while the spinunrestricted KS reference can be designed as an eigenfunction of $\hat{S}_{z}$ it is not necessarily an eigenfunction of $\hat{S}^{2}$. Regarding DFT, Perdew et al. have pointed out that some degree of spin contamination is good because the KS reference is not the true wavefunction [20]. Spin polarization is generated straightforwardly in spin-unrestricted calculations but can be severely over- or under-estimated. Singlereference KS methods with approximate functionals are often not suited to represent degenerate states. The calculation then results in breaking of spin or spatial symmetry of the wavefunction, or both. Projection techniques can be used to restore lost symmetries.

In the absence of orbital degeneracy, the components of a pseudo-spin doublet can often be treated reasonably well by standard spin-unrestricted KS methods for the purpose of calculating EPR spin Hamiltonian parameters. In a SR or NR framework, the $g$-factors then simply become equal to $g_{e}$, while the isotropic average of the HFC matrix, the isotropic HFC constant, is calculated from averaging $g_{K} \beta_{N}\left\langle\psi\left|\hat{F}_{K k}\right| \psi\right\rangle$ over $k$, with $|\psi\rangle$ being the $\mathscr{M}_{\mathscr{S}}=+1 / 2$ component of the doublet. There is also extensive literature on utilizing the same expectation value approach within single-reference correlated wavefunction methods. Extensions to treat cases with $S>(1 / 2)$, and ways for additional inclusion of SO coupling via first-order perturbation theory, have been devised. The reader can find details in References 21-27 and citations to original literature provided therein.

Some of the KS methods that are currently in use for relativistic EPR parameter calculations with SO coupling being included variationally [28-30] employ two different approaches. The first utilizes a variant of Equation (0.13), but within a single-electron framework where the many-electron wavefunctions are replaced by one-electron orbitals. In the second approach, three separate SCF cycles are typically performed, with different quantization axes of spin, magnetic moment, or total angular momentum, and the quantization axis is identified with the directional index ' $k$ ' of Equations (0.10) and (0.13).

The first approach $[28,30]$ as it was devised and implemented in a twocomponent relativistic form is quasi spin-restricted and limited to Kramers doublets. An SCF calculation is performed with the unpaired electron distributed over two degenerate frontier orbitals, with occupations of $1 / 2$ each. In the absence of SO coupling, these would be an $\alpha$ and $\beta$ spin pair of orbitals with identical spatial components. The method has some resemblance to restricted open-shell HF (ROHF) but is not the same. After the SCF step, one of these orbitals, say $\varphi$, is chosen to represent the component $\phi_{1}$ of the Kramers pair of orbitals. Its conjugate $\phi_{2}$ is then constructed from $\phi_{1}$. Written explicitly in terms of real (R) and imaginary (I) parts of the two spin components of the SCF orbital $\varphi$, the Kramers pair is 


$$
\begin{aligned}
\phi_{1} & =\left(\begin{array}{c}
\varphi_{\alpha}^{R} \\
\varphi_{\beta}^{R}
\end{array}\right)+i\left(\begin{array}{c}
\varphi_{\alpha}^{I} \\
\varphi_{\beta}^{I}
\end{array}\right) \\
\phi_{2} & =\left(\begin{array}{c}
-\varphi_{\beta}^{R} \\
\varphi_{\alpha}^{R}
\end{array}\right)+i\left(\begin{array}{c}
\varphi_{\beta}^{I} \\
-\varphi_{\alpha}^{I}
\end{array}\right)
\end{aligned}
$$

For calculations of $g$-factors, the matrix elements of the Zeeman operator are then calculated as in Equation (0.13), but with the orbital pair $\left\{\phi_{1}, \phi_{2}\right\}$, and then processed similar to Equations (0.14), (0.15) to yield the $g$-factor. Alternatively, the $h_{k}^{\prime}$ of Equation (0.13) are directly identified with the $h_{k}$ terms of Equation (0.10), and the $g$-matrix elements can be extracted from the calculation results without detour via G. HFC matrix elements can be calculated in an analogous way. However, due to the lack of spin polarization the performance of the quasi-restricted approach is unsatisfactory for the latter. The performance for $g$-factors has frequently been satisfactory.

Regarding the 'three SCF cycles' techniques, van Wüllen and co-workers have provided a justification for their use in KS calculations [31]. The approach is illustrated for HFC [30]: The expectation value of the HFC part of the EPR spin Hamiltonian taken with a Kohn-Sham determinant calculated with a spin-quantization axis $\boldsymbol{u}$

$$
E(\boldsymbol{u})=\sum_{i} n_{i}\left\langle\varphi_{i}^{\boldsymbol{u}}\left|\boldsymbol{I}_{K} \cdot \mathbf{a} \cdot \hat{\mathscr{S}}\right| \varphi_{i}^{\boldsymbol{u}}\right\rangle=\sum_{k, l} a_{k l} I_{K k} \sum_{i} n_{i}\left\langle\varphi_{i}^{\boldsymbol{u}}\left|\hat{\mathscr{S}}_{l}\right| \varphi_{i}^{\boldsymbol{u}}\right\rangle
$$

with $k, l \in\{x, y, z\}$. The $\varphi_{i}^{u}$ are assumed to be orbitals obtained from a 'generalized collinear' KS calculation with selected spin-quantization axis $\boldsymbol{u}$, and the $n_{i}$ are the occupation numbers. Assume next that $\boldsymbol{u}$ is along the Cartesian direction $k$, that the orbitals are $\hat{S}_{k}$ eigenfunctions, that the electron spin $S$ is the same as the pseudo-spin $\mathscr{S}$, and that the KS determinant is a solution corresponding to $\left\langle S_{k}\right\rangle=M_{S}=S=\mathscr{S}$. One then finds $\sum_{i} n_{i}\left\langle\varphi_{i}^{k}\left|\hat{S}_{l}\right| \varphi_{i}^{k}\right\rangle=\mathscr{S} \delta_{k l}$, such that

$$
E(k)=\mathscr{S} \sum_{l} a_{k l} I_{K l}
$$

Instead, calculate an analogous expectation value, but this time with the QM hyperfine operator $g_{K} \beta_{N} \hat{\boldsymbol{F}} \cdot \boldsymbol{I}_{K}$ and with the actual relativistic generalized-collinear two-component KS orbitals,

$$
E(k)=g_{K} \beta_{N} \sum_{l} \sum_{i} n_{i}\left\langle\varphi_{i}^{k}\left|\hat{F}_{K l}\right| \varphi_{i}^{k}\right\rangle I_{K l}
$$

One can now map the result (0.20) for the pseudo-spin onto the result (0.21) calculated by $\mathrm{KS}$, which gives

$$
a_{k l}=\frac{g_{K} \beta_{N}}{\mathscr{S}} \sum_{i} n_{i}\left\langle\varphi_{i}^{k}\left|\hat{F}_{K l}\right| \varphi_{i}^{k}\right\rangle
$$


An analogous approach is possible for calculations of $g$-factors, which gives with the QM Zeeman operator $-\beta_{e} \hat{\boldsymbol{\mu}} \cdot \boldsymbol{B}$

$$
g_{k l}=-\frac{1}{\mathscr{S}} \sum_{i} n_{i}\left\langle\varphi_{i}^{k}\left|\hat{\mu}_{l}\right| \varphi_{i}^{k}\right\rangle
$$

Both for the Zeeman and HFC matrices, one can form the rank-2 tensors $\mathbf{A}_{K}$ and $\mathbf{G}$ afterwards and diagonalize them in order to obtain the PAS. As with variational wavefunction methods, GIAO basis sets are sometimes employed in order to generate Zeeman coupling matrices that are strictly origin invariant.

Within the generalized-collinear KS framework, it is also possible to obtain elements of the ZFS tensor d, from the magnetic anisotropy of the KS energy with respect to the spin quantization axis $\boldsymbol{u}$. With the pseudo-spin Hamiltonian and a $\mathscr{M}_{\mathscr{S}}=\mathscr{S}$ pseudo-spin eigenfunction, one obtains

$$
E^{\mathrm{ZFS}}(\boldsymbol{u})=\mathscr{S}\left(\mathscr{S}-\frac{1}{2}\right) \boldsymbol{u} \cdot \mathbf{d} \cdot \boldsymbol{u}
$$

As for the other parts of the EPR spin Hamiltonian, the result of a QM calculation of $E(\boldsymbol{u})$ for different directions of $\boldsymbol{u}$ can then be mapped onto Equation (0.24). The approach was first introduced in References 31,32, where the reader can find comments regarding some subtleties leading to the $\mathscr{S}(\mathscr{S}-1 / 2)$ factor instead of $\mathscr{S}^{2}$. For weak SO coupling, $E(\boldsymbol{u})$ can also be calculated by perturbation theory. In this case, the 'sum over states' (SOS) - like equation (0.8) can be interpreted as the result of a double perturbation of the energy by SO coupling and the dipolar ZFS interaction, and a KS coupled-perturbed analog can be devised instead. For details, see Reference 31. In cases where SO coupling dominates the ZFS, and for spin triplets, there is another KS route: Starting with a closed-shell reference state, one calculates energy differences between the reference and a triplet state of interest by time-dependent linear response ('time-dependent DFT') within a framework that includes SO coupling variationally or as a perturbation.

\subsubsection{Operators for the Zeeman and HFC interactions}

In principle, the Zeeman and hyperfine operators that are used in QM calculations of EPR parameters should match the Hamiltonian used for calculating the wavefunctions or KS orbitals in order to avoid picture-change errors. For further details, the reader is referred to the chapters in this Handbook that are concerned with details of relativistic calculations of NMR parameters within various relativistic frameworks, because derivatives of the Zeeman and hyperfine operators with respect to the external field components and the nuclear spin magnetic moment components, respectively, are needed for those calculations. In order to render this chapter somewhat self-contained, for illustration, the Zeeman and hyperfine one-electron operators are provided here for the NR case, for the two-component zeroth-order regular approx- 
imation (ZORA), and for the four-component case in its standard notation where diamagnetism is not explicit. For brevity, field-dependent two-electron operators are not listed.

Assuming point nuclei for the hyperfine terms, the gauge origin for the external field coinciding with the coordinate origin, and Coulomb gauge for the nuclear and external vector potential, the nonrelativistic Zeeman $(Z)$ and HFC one-electron operators read

$$
\begin{aligned}
\mathrm{NR}: \hat{h}^{Z} & =\frac{\beta_{e}}{\hbar}[(\boldsymbol{r} \times \hat{\boldsymbol{p}})+\hbar \boldsymbol{\sigma}] \cdot \boldsymbol{B} \\
& =\beta_{e}[\hat{\boldsymbol{L}}+2 \hat{\boldsymbol{S}}] \cdot \boldsymbol{B} \\
\hat{h}_{K}^{\mathrm{HFC}} & =\frac{2 \beta_{e}}{\hbar} \frac{\mu_{0}}{4 \pi}\left[\frac{\boldsymbol{r}_{K}}{r_{K}^{3}} \times \hat{\boldsymbol{p}}\right] \cdot \boldsymbol{\mu}_{K} \\
& +\beta_{e} \frac{\mu_{0}}{4 \pi}\left[\boldsymbol{\sigma} \cdot\left\{\boldsymbol{\mu}_{K}\left(\boldsymbol{\nabla} \cdot \frac{\boldsymbol{r}_{K}}{r_{K}^{3}}\right)-\left(\boldsymbol{\mu}_{K} \cdot \boldsymbol{\nabla}\right) \frac{\boldsymbol{r}_{K}}{r_{K}^{3}}\right\}\right]
\end{aligned}
$$

Curly brackets, $\{\cdots\}$, in the operator expressions indicate that derivatives are only taken inside the operator, not of functions to its right hand side. As elsewhere in this chapter, $\boldsymbol{\mu}_{K}=g_{K} \beta_{N} \boldsymbol{I}_{K}$. Further, $\boldsymbol{r}_{K}$ is the electron-nucleus distance vector and $r_{K}$ its length. The Zeeman operator is a sum of contributions from orbital and spin angular momentum. Likewise, in the hyperfine operator there is the 'Paramagnetic nuclear Spin - electron Orbital' (PSO) term in Equation (0.25b) which is independent of the electron spin, and there is the electron spin dependent sum of the Fermi contact (FC) and spin dipole (SD) operators in Equation (0.25c). The usual expressions for the FC and SD operators are obtained by taking the derivatives of $\boldsymbol{r}_{K} / r_{K}^{3}$, which gives

$$
\begin{aligned}
\mathrm{NR}: & \hat{h}_{K}^{\mathrm{FC}}=\beta_{e} \frac{\mu_{0}}{4 \pi} \frac{8 \pi}{3} \delta\left(\boldsymbol{r}_{K}\right) \boldsymbol{\sigma} \cdot \boldsymbol{\mu}_{K} \\
\hat{h}_{K}^{\mathrm{SD}} & =\beta_{e} \frac{\mu_{0}}{4 \pi} \frac{3\left(\boldsymbol{\sigma} \cdot \boldsymbol{r}_{K}\right)\left(\boldsymbol{\mu}_{K} \cdot \boldsymbol{r}_{K}\right)}{r_{K}^{5}}
\end{aligned}
$$

The 'contact' part of the name of the FC operator refers to the presence of the Dirac $\delta$-distribution.

Due to the fact that code for calculating matrix elements of these operators with Gaussian-type atomic orbital (AO) basis functions is rather widely available, nonrelativistic operators are sometimes used in relativistic calculations of EPR parameters. For the Zeeman operator, the relativistic corrections from the operator are likely small because it samples the valence and outer regions of light and heavy atoms. The hyperfine operators are to be used in relativistic calculations only with caution, because of the singular behavior evident from Equations $(0.26 \mathrm{a}, 0.26 \mathrm{~b})$. Due to their local nature it is possible to use them for light nuclei in a system that also contains heavy elements, because then the relativistic effects are not generated around the nucleus for which the HFC is calculated. It may also be possible to generate estimates of a heavy-element HFC if the relevant orbitals have high angular momentum 
and the HFC is dominated by the PSO mechanism. It is certainly not physically meaningful to use the nonrelativistic hyperfine operators in other relativistic scenarios such as HFC tensors of heavy alkali metal atoms or for small radicals containing mercury (see Section 0.5).

When adopting the two-component ZORA framework, the operators relevant for the Zeeman and HFC interactions read

$$
\text { ZORA : } \begin{aligned}
\hat{h}^{Z} & =\frac{\beta_{e}}{2 \hbar}[\mathcal{K}(\boldsymbol{r} \times \hat{\boldsymbol{p}})+(\boldsymbol{r} \times \hat{\boldsymbol{p}}) \mathcal{K}] \cdot \boldsymbol{B} \\
& +\frac{\beta_{e}}{2} \boldsymbol{\sigma} \cdot\{\boldsymbol{B}(\boldsymbol{\nabla} \cdot \mathcal{K} \boldsymbol{r})-(\boldsymbol{B} \cdot \boldsymbol{\nabla}) \mathcal{K} \boldsymbol{r}\} \\
\hat{h}_{K}^{\mathrm{HFC}} & =\frac{\beta_{e}}{\hbar} \frac{\mu_{0}}{4 \pi}\left[\mathcal{K} \frac{\boldsymbol{r}_{K}}{r_{K}^{3}} \times \hat{\boldsymbol{p}}+\frac{\boldsymbol{r}_{K}}{r_{K}^{3}} \times \hat{\boldsymbol{p}} \mathcal{K}\right] \cdot \boldsymbol{\mu}_{K} \\
& +\frac{\beta_{e} \mu_{0}}{4 \pi}\left[\boldsymbol{\sigma} \cdot\left\{\boldsymbol{\mu}_{K}\left(\boldsymbol{\nabla} \cdot \frac{\mathcal{K} \boldsymbol{r}_{K}}{r_{K}^{3}}\right)-\left(\boldsymbol{\mu}_{K} \cdot \boldsymbol{\nabla}\right) \frac{\mathcal{K} \boldsymbol{r}_{K}}{r_{K}^{3}}\right\}\right]
\end{aligned}
$$

The function $\mathcal{K}=2 m_{e} c^{2} /\left(2 m_{e} c^{2}-V\right)$ is a 'relativistic kinematic factor' that typically shows up in equations derived within the ZORA framework. Formally, the NR limit is obtained for $\mathcal{K} \rightarrow 1$. In this case, $(0.27 \mathrm{a})$ becomes the orbital Zeeman $(\mathrm{OZ})$ operator, $(0.27 \mathrm{~b})$ becomes spin Zeeman, $(0.27 \mathrm{c})$ becomes PSO, and $(0.27 \mathrm{~d})$ becomes FC+SD. It therefore makes sense to adopt the same terminology with twocomponent methods such as ZORA, Douglas Kroll Hess (DKH) beyond first order, and other approximate or formally exact two-component methods that afford operators of similar structure. In the vicinity of heavy nuclei, $\mathcal{K}$ is very different from unity which generates the desired relativistic effects. It is noted that for point nuclei with a charge below 118 there is no 'contact' term (i.e. a delta distribution) [33], because it is suppressed by $\mathcal{K} \rightarrow 0$ for $r_{K} \rightarrow 0$ in the operator. Above 118 the ZORA method breaks down for hyperfine effects because the singularities of $s_{1 / 2}$ and $p_{1 / 2}$ orbitals at the nucleus become too strong. [33] With extended nuclei, the behavior is more realistic.

The one-electron Zeeman and hyperfine operators in the four-component (Dirac) framework involve the $4 \times 4$ Dirac $\boldsymbol{\alpha}$ matrices:

$$
\begin{aligned}
\text { Dirac }: \quad \hat{h}^{Z} & =\frac{c e}{2} \boldsymbol{r} \times \boldsymbol{\alpha} \cdot \boldsymbol{B} \\
\hat{h}_{K}^{\mathrm{HFC}} & =\frac{c e \mu_{0}}{4 \pi} \frac{\boldsymbol{r}_{K}}{r_{K}^{3}} \times \boldsymbol{\alpha} \cdot \boldsymbol{\mu}_{K}
\end{aligned}
$$

Unlike the NR and two-component versions, the operators do not explicitly include derivative terms. However, the derivative terms are implicitly contained in the formalism because of the relation between the large (upper) and small (lower) components of the electronic wavefunctions or orbitals.

For HFC that is nominated by $s$ orbitals (heavy alkali metals and $\mathrm{Hg}$ in particular), finite nucleus effects can be large. There are different ways to treat finite nuclear volume effects [34]. Due to the ubiquity of Gaussian-type basis functions in quantum chemical calculations, the spherical Gaussian nuclear model is in widespread 
use. Here, the charge distribution $\rho_{K}$ of nucleus $A$ is 'smeared out' by a Gaussian function as

$$
\rho_{K}(\boldsymbol{R})=Z_{K}\left(\frac{\xi_{K}}{\pi}\right)^{3 / 2} e^{-\xi_{K}\left|\boldsymbol{R}-\boldsymbol{R}_{K}\right|^{2}}
$$

The exponent $\zeta_{K}$ is inversely proportional to the mean square radius $\left\langle R_{K}^{2}\right\rangle$ of the nucleus:

$$
\zeta_{K}=\frac{3}{2\left\langle R_{K}^{2}\right\rangle}
$$

The root mean square nuclear radius is, in turn, related to the nuclear mass $M_{K}$ (in amu) as follows:

$$
\left\langle R_{K}^{2}\right\rangle^{1 / 2}=\left(0.863 M_{K}^{1 / 3}+0.570\right) \mathrm{fm}
$$

The electron-nucleus attraction term for nucleus $A$ with charge $Z_{K}$ in the Hamiltonian for point nuclei,

$$
V_{K}^{\text {point }}=-\frac{1}{4 \pi \varepsilon_{0}} \frac{Z_{K}}{r_{K}}
$$

changes to

$$
V_{K}^{\text {gauss. }}=-\frac{1}{4 \pi \varepsilon_{0}} \frac{Z_{K}}{r_{K}} P\left(1 / 2, \tilde{r}_{K}^{2}\right)
$$

with $\tilde{r}_{K}^{2}=\zeta_{K} r_{K}^{2}$. Further,

$$
P(a, x)=\frac{1}{\Gamma(a)} \int_{0}^{x} t^{a-1} e^{-t}
$$

is the lower incomplete Gamma function ratio. Assuming as a first approximation that the magnetization density of the nucleus can also be described by a spherical Gaussian, the vector potential for a point nucleus,

$$
\boldsymbol{A}_{K}^{\text {point }}=\frac{\mu_{0}}{4 \pi} \frac{\boldsymbol{\mu}_{K} \times \boldsymbol{r}_{K}}{r_{K}^{3}}
$$

changes to

$$
\boldsymbol{A}_{K}^{\text {gauss. }}=\frac{\mu_{0}}{4 \pi} \frac{\boldsymbol{\mu}_{K} \times \boldsymbol{r}_{K}}{r_{K}^{3}} P\left(3 / 2, \tilde{r}_{K}^{2}\right)
$$

The presence of the incomplete Gamma function terms in the expressions serves to dampen the inverse powers of $r_{K}$ such that the resulting potential and vector potential remain finite as $r_{K} \rightarrow 0$. In calculations, there are two effects: The first one is via the potential $(0.33 \mathrm{~b})$ and affects the electron spin and orbital magnetizations around the nucleus. The second one is the modification of the hyperfine operators 
by $(0.35 b)$. In combination, they tend to reduce the magnitude of hyperfine coupling constants.

\subsection{Higher-order EPR parameters, and mapping of ab-initio to pseudo-spin functions}

This section focuses on ZFS and the Zeeman interaction as examples. The HFC can be treated in an analogous fashion as the Zeeman interaction as far as higher order pseudo-spin Hamiltonian terms are concerned. For a unified formalism and examples see Reference 35.

The larger the pseudo-spin $\mathscr{S}$, the more degrees of freedom there are. Higherorder of spin operators are then added to the spin Hamiltonian to describe the supplementary degrees of freedom. Higher orders include terms with polynomials of order $l, m$ and $n$ in the components of $\hat{\mathscr{S}}, \boldsymbol{B}$ and $\boldsymbol{I}$ respectively, symbolically denoted here as a term of order $\mathscr{S}^{l} B^{m} I^{n}$ where $l, m$ and $n$ are non-negative integers and $l+m+n$ is even to preserve time even parity of the Hamiltonian. An exception concerns the description of non-Kramers doublets. This point is presented in Section 0.5 . The expansion is limited to $l \leq 2 \mathscr{S}$ since all matrix elements of the operators with $l>2 \mathscr{S}$ are zero. The ZFS term corresponds to $m=n=0$

$$
\hat{\mathcal{H}}_{S}^{Z F S}=\hat{\mathcal{H}}_{(2)}^{Z F S}+\hat{\mathcal{H}}_{(4)}^{Z F S}+\cdots
$$

where $\hat{\mathcal{H}}_{(l)}^{Z F S}$ is a term of order $l$ even in $\mathscr{S}$. The term linear in the magnetic field, with $m=1$ and $n=0$ is the Zeeman term

$$
\begin{aligned}
\hat{\mathcal{H}}_{S}^{Z} & =\hat{\mathcal{H}}_{(1)}^{Z}+\hat{\mathcal{H}}_{(3)}^{Z}+\cdots \\
& =\beta_{e}\left(\hat{\boldsymbol{\mu}}_{(1)}+\hat{\boldsymbol{\mu}}_{(3)}+\cdots\right) \cdot \boldsymbol{B}
\end{aligned}
$$

where $\hat{\boldsymbol{\mu}}_{(l)}$ is a term of order $l$ odd in $\mathscr{S}$. The next term with $m=2$ describes the quadratic contribution in the magnetic field. This term is usually negligible due the smallness of the magnetic interaction. [36]

According to the irreducible tensor operator decomposition, the preceding terms can be written as

$$
\hat{\mathcal{H}}_{(l)}^{Z F S}=\sum_{m=-l}^{l} a_{l, m} \mathcal{T}_{l, m}(\hat{\mathscr{S}})
$$

and

$$
\hat{\mu}_{(l)}^{u}=\sum_{m=-l}^{l} b_{l, m}^{u} \mathcal{T}_{l, m}(\hat{\mathscr{S}})
$$

where $\hat{\mu}_{(l)}^{u}$ is the component of $\hat{\boldsymbol{\mu}}_{l}$ in direction $\boldsymbol{u}$ and $\mathcal{T}_{l m}$ are the tesseral combinations of the spherical-tensor operators $T_{l, m}$ 


$$
\begin{aligned}
& \mathcal{T}_{l, m}(\hat{\mathscr{S}})=\frac{1}{\sqrt{2}}\left[(-1)^{m} T_{l, m}(\hat{\mathscr{S}})+T_{l,-m}(\hat{\mathscr{S}})\right] \\
& \mathcal{T}_{l,-m}(\hat{\mathscr{S}})=\frac{i}{\sqrt{2}}\left[(-1)^{m+1} T_{l, m}(\hat{\mathscr{S}})+T_{l,-m}(\hat{\mathscr{S}})\right]
\end{aligned}
$$

with $0 \leq m \leq l$. Equation 0.39 becomes for $l=1$

$$
\hat{\mu}_{(1)}^{u}=b_{1,1}^{u} \hat{\mathscr{S}}_{x}+b_{1,-1}^{u} \hat{\mathscr{S}}_{y}+b_{1,0}^{u} \hat{\mathscr{S}}_{z}
$$

with $u=x, y, z$. This defines nine parameters $b_{1, m}^{u}$ corresponding to the elements of the $\mathrm{g}$ matrix. Equation 0.41 corresponds to the first term of Equation 0.6 and appears for all values of $\mathscr{S} \geq 1 / 2$. The third term of Equation 0.6 appears for $\mathscr{S} \geq 1$

$$
\begin{aligned}
\hat{\mathcal{H}}_{(2)}^{Z F S}= & a_{2,2} \frac{1}{\sqrt{2}}\left(\hat{\mathscr{S}}_{x}^{2}-\hat{\mathscr{S}}_{y}^{2}\right)+a_{2,-2} \frac{1}{\sqrt{2}}\left(\hat{\mathscr{S}}_{x} \hat{\mathscr{S}}_{y}+\hat{\mathscr{S}}_{y} \hat{\mathscr{S}}_{x}\right)+a_{2,1} \frac{1}{\sqrt{2}}\left(\hat{\mathscr{S}}_{x} \hat{\mathscr{S}}_{z}+\hat{\mathscr{S}}_{z} \hat{\mathscr{S}}_{x}\right) \\
& +a_{2,-1} \frac{1}{\sqrt{2}}\left(\hat{\mathscr{S}}_{y} \hat{\mathscr{S}}_{z}+\hat{\mathscr{S}}_{z} \hat{\mathscr{S}}_{y}\right)+a_{2,0} \frac{1}{\sqrt{6}}\left(2 \hat{\mathscr{S}}_{z}^{2}-\hat{\mathscr{S}}_{x}^{2}-\hat{\mathscr{S}}_{y}^{2}\right)
\end{aligned}
$$

The five parameters $a_{2, m}$ defines the symmetric and traceless $\mathbf{d}$ tensor. The cubic term in $\mathscr{S}$ contributes to the Zeeman interaction for $\mathscr{S} \geq 3 / 2$,

$$
\hat{\mu}_{(3)}^{u}=\sum_{m=-3}^{3} b_{3, m}^{u} \mathcal{T}_{3, m}(\hat{\mathscr{S}})
$$

All $\mathcal{T}_{3, m}(\hat{\mathscr{S}})$ can be expressed as a product of 3 spin components $\hat{\mathscr{S}}_{u}$ defining a third-rank tensor $\mathbf{g}^{\prime}$.

$$
\hat{\mathcal{H}}_{(3)}^{Z}=\beta_{e} \boldsymbol{B} \cdot \mathbf{g}^{\prime} \cdot \hat{\mathscr{S}}^{3}
$$

where $\mathbf{g}^{\prime}$ is a third-rank tensor.

In the case of weak SO coupling, the $|\lambda, a\rangle$ wave functions correspond closely to the SR components $\left|\lambda^{\prime}, a\right\rangle$. Without SO coupling, the $\left|\lambda^{\prime}, a\right\rangle$ functions are the $2 S+1$ spin components of the real spin and degenerate. These $\left|\lambda^{\prime}, M\right\rangle$ behave properly under all spin operations, time inversion and spatial symmetries of the molecule and can be assigned to the pseudo-spin states $\left|\lambda^{\prime}, M_{S}\right\rangle \equiv\left|\mathscr{S}, \mathscr{M}_{S}\right\rangle$. As it has been shown in Section 0.2.1, the spin Hamiltonian parameters can be calculated in this case by a perturbative approach using wave functions which do not include SO effects.

In the case where SO coupling is included in the QM calculation, the assignment of the $|\lambda, a\rangle$ functions to pseudo-spin functions becomes more difficult. There are currently two types of methods to calculate the spin Hamiltonian parameters from the $E_{a}$ and the three matrices $\boldsymbol{\mu}^{u}$ of the electron magnetic moment represented in the basis of $|\lambda, a\rangle$ calculated by the $a b$-initio methods. i) either by projecting the Zeeman matrices using the Irreducible Tensor Operators algebra. [10] Since these latter operators form a basis of orthogonal and linearly independent matrices, each matrix has a unique expansion in this basis set. ii) or by mapping the matrix elements of the real and pseudo-spin matrices one by one once the correspondence between the real and pseudo-spin states is performed.

For the projection technique, one considers first that the term linear in $\mathscr{S}$ is the dominant one in the $\hat{\mathcal{H}}_{S}^{Z}$ operator and the tensor $\mathbf{G}=\mathbf{g} \mathbf{g}^{T}$ can be calculated as [11] 


$$
\mathbf{G}=\frac{6}{\mathscr{S}(\mathscr{S}+1)(2 \mathscr{S}+1)} \mathcal{A}
$$

where the tensor $\mathcal{A}$ (not to be confused with $\mathbf{A}$ of Equation (0.17)) is defined as

$$
\mathcal{A}_{k, l}=\frac{1}{2} \operatorname{tr}\left(\boldsymbol{\mu}^{k} \boldsymbol{\mu}^{l}\right)
$$

The diagonalization of $\mathbf{G}$ provides the absolute values of the $\mathrm{g}$ factors $g_{i}= \pm \sqrt{G_{i}}$ with $i=X, Y, Z$. Eq. 0.45 is equivalent to Eq. 0.15 in the case $\mathscr{S}=1 / 2$.

The matching technique consists in rotating the three matrices $\boldsymbol{\mu}^{k}(k=x, y, z)$

i) in the Euclidean space of spatial coordinates $\mathbf{R}_{k, l}$

$$
\boldsymbol{\mu}^{l^{\prime}}=\sum_{k} \mathbf{R}_{k, l^{\prime}} \boldsymbol{\mu}^{k} \quad \text { with } \quad k=x, y, z \quad \text { and } \quad l^{\prime}=x^{\prime}, y^{\prime}, z^{\prime}
$$

where $\mathbf{R}$ is a $3 * 3$ rotation matrix of the Cartesian coordinates.

ii $)$ in the Hilbert space generated by $|\lambda, a\rangle(a=1,2 \mathscr{S}+1)$.

$$
\left(\boldsymbol{\mu}^{k}\right)^{\prime}=\mathcal{R}^{\dagger} \cdot \boldsymbol{\mu}^{k} \cdot \mathcal{R} \text { for all } k=x, y, z
$$

where $\mathcal{R}$ is a rotation in the $(1+2 \mathscr{S})^{2}$ Hilbert space

These rotations are performed in order to put the three matrices $\boldsymbol{\mu}^{k}(k=x, y, z)$ to suit the matrices of the spin Hamiltonian. The rotations in coordinate space may rotate the real space in the principal axis of the $\mathrm{D}$ or $\mathcal{A}$ for example. Then the rotations in the Hilbert space of wave functions may diagonalize the $\boldsymbol{\mu}^{Z}$ matrix, make $\boldsymbol{\mu}^{X}$ real and $\boldsymbol{\mu}^{Y}$ imaginary. No information is lost during these transformations. In the final form, one can fit the spin Hamiltonian parameters on the matrix elements of the $\boldsymbol{\mu}^{\prime k}\left(k=x^{\prime}, y^{\prime}, z^{\prime}\right)$ matrices. The deviation through the fitting procedure can be evaluated and scores the propensity of the model to reproduce the ab-initio data. But this procedure need some symmetry in order to find the proper rotations.

For the calculation of the ZFS parameters, one needs the assignment of the combination of the $|\lambda, a\rangle$ to the pseudo-spin states $|\mathscr{S}, \mathscr{M}\rangle$. One must find a rotation $\mathcal{R}$ in the model space such the transformed wavefunctions fulfill the time inversion properties $\hat{\Theta}|\lambda, \mathscr{M}\rangle=( \pm 1)^{(\mathscr{S}-\mathscr{M})}|\lambda,-\mathscr{M}\rangle$. The real Hamiltonian, including the interactions attributed to the ZFS, is diagonal in the basis of the $|\lambda, a\rangle$ if SO coupling and the dipolar two-electron spin-spin interaction are included variationally in the ground state. In the new basis set, it becomes

$$
\mathbf{H}^{\mathrm{ZFS}}=\mathcal{R}^{\dagger} \cdot \mathbf{E} \cdot \mathcal{R}
$$

where $\mathbf{E}$ is the diagonal matrix with $\mathbf{E}_{a, b}=E_{a} \delta_{a, b}$. Then, these matrix elements match the matrix elements of the pseudo-spin matrix $\mathbf{H}_{\mathscr{M}, \mathscr{M}^{\prime}}^{\mathrm{ZFS}}=$ $\left\langle\mathscr{S}, \mathscr{M}\left|\hat{\mathcal{H}}_{S}^{Z F S}\right| \mathscr{S}, \mathscr{M}\right\rangle$

If SO coupling is weak, the $|\lambda, a\rangle$ derives mostly of a pure spin state ${ }^{2 S+1} \Gamma$ with components $\left|\lambda^{\prime}, M\right\rangle$ where $M$ is the projection on the quantification axis of the real spin. This assignment is easily performed using the effective Hamiltonian 
technique [37] briefly outlined in Sec. 0.2.1.

$$
\mathcal{P}|\lambda, a\rangle=\sum_{M} C_{i, M}\left|\lambda^{\prime}, M\right\rangle
$$

where $\mathcal{P}$ is the projector on the pure spin space. The effective Hamiltonian in this target space is

$$
\mathbf{H}_{\text {eff }}^{Z F S}=\mathcal{C}^{-1} \cdot \mathbf{E} \cdot \mathcal{C}
$$

where $\mathcal{C}_{i, M}=C_{i, M} \cdot \mathcal{C}_{i, M}$ is not an orthogonal matrix since it is a projector. The wave functions $\left|\lambda^{\prime}, M\right\rangle$ satisfy all the properties of transformations of spin with time inversion and spin operators since they are eigenfunctions for a real spin. Equations 0.49 and 0.51 are closely related. In the case of weak SO coupling, $\mathbf{C}$ is close to being orthogonal and the effective Hamiltonian procedure is a convenient way to obtain the rotation $\mathcal{R}$, eventually using an orthogonalization procedure. This procedure is applicable for all values of $\mathscr{S}$ while for large values of $\mathscr{S}$ the determination of $\mathcal{R}$ becomes more complicated due to the increase of the number of degrees of freedom.

The spin Hamiltonian is designed in order to reduce as much as possible the number of parameters to fit the EPR spectra. In the case of high $\mathscr{S}$, many of the spin Hamiltonian parameters are negligible. In the case of weak SO coupling, the Zeeman interaction is almost isotropic and the magnetic anisotropy arises from the ZFS term. The determination of the ZFS tensor is then the key step of the fitting.

In the case of $\mathscr{S}=1$, without any spatial symmetry, the three $|\lambda, a\rangle$ are not degenerate and are not magnetic to first order $\left\langle\lambda, a\left|\hat{\boldsymbol{\mu}}_{u}\right| \lambda, a\right\rangle=0$. Magnetic properties arise from the off-diagonal matrix elements. The spin Hamiltonian expressed in the principal axis of the $\mathbf{d}$ tensor with an isotropic Zeeman interaction writes

$$
\begin{aligned}
\hat{\mathcal{H}}_{S} & =\beta_{e} g \boldsymbol{B} \cdot \hat{\mathscr{S}}+d_{X} \hat{\mathscr{S}}_{X}^{2}+d_{Y} \hat{\mathscr{S}}_{Y}^{2}+d_{Z} \hat{\mathscr{S}}_{Z}^{2} \\
& =\beta_{e} g \boldsymbol{B} \cdot \hat{\mathscr{S}}+D\left(\hat{\mathscr{S}}_{Z}^{2}-\frac{1}{3} \mathscr{S}(\mathscr{S}+1)\right)+E\left(\hat{\mathscr{S}}_{X}^{2}-\hat{\mathscr{S}}_{Y}^{2}\right)
\end{aligned}
$$

In the basis set

$\left\{\left|0_{X}\right\rangle=\frac{1}{\sqrt{2}}(-|1,1\rangle+|1,-1\rangle),\left|0_{Y}\right\rangle=\frac{i}{\sqrt{2}}(|1,1\rangle+|1,-1\rangle),\left|0_{Z}\right\rangle=|1,0\rangle\right\}$ where $\left|0_{u}\right\rangle$ is the spin state with $\mathscr{M}=0$ in the direction $u$

$$
\begin{array}{c|ccc}
\hat{\mathcal{H}}_{S} & \left|0_{X}\right\rangle & \left|0_{Y}\right\rangle & \left|0_{Z}\right\rangle \\
\hline\left\langle 0_{X}\right| & \frac{1}{3} D-E & -i \beta_{e} g B_{Z} & i \beta_{e} g B_{Y} \\
\left\langle 0_{Y}\right| & i \beta_{e} g B_{Z} & \frac{1}{3} D+E & -i \beta_{e} g B_{X} \\
\left\langle 0_{Z}\right| & -i \beta_{e} g B_{Y} & i \beta_{e} g B_{X} & -\frac{2}{3} D
\end{array}
$$

Fig. 0.1 represents the variation of the energy of the three states as a function of $B$ for the three directions $X, Y$ and $Z$ for $g=2, D=10 \mathrm{~cm}^{-1}$ and $E=1$ $\mathrm{cm}^{-1}$. The largest magnetization (slope of the $E=f(B)$ curve) is obtained for $\left|0_{X}\right\rangle$ and $\left|0_{Y}\right\rangle$ when the field is applied along $Z$ since $\left|0_{X}\right\rangle$ and $\left|0_{Y}\right\rangle$ are the closest and the Zeeman interaction couples them in this direction. When $D<0$, these two states are lower than $\left|0_{Z}\right\rangle$ and the ground state is magnetic along $Z$; smaller is $E$, 

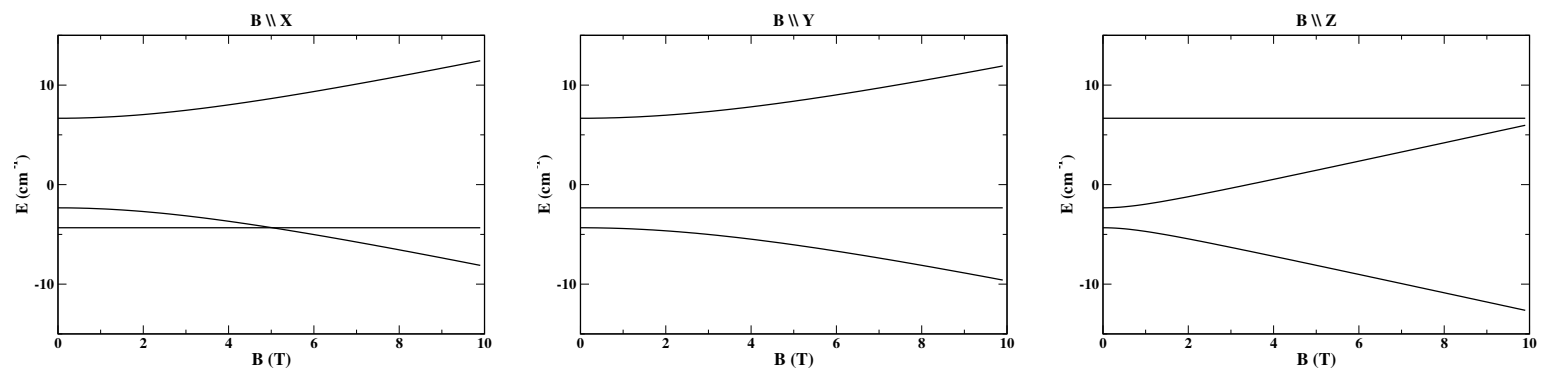

Fig. 0.1 Energy versus magnetic field for $\mathscr{S}=1$ as solution of Eq. 0.53 for $g=2, D=-10$ $\mathrm{cm}^{-1}$ and $E=1 \mathrm{~cm}^{-1}$

larger the magnetic interaction between these states is. This corresponds to an axial magnetization in direction $Z$.

The spin Hamiltonian of Equation 0.52 is widely used for $\mathscr{S}=3 / 2$ systems as well. It will be shown in Section 0.5 that the cubic term in $\mathscr{S}$ is negligible in the case of weak SO coupling. In the basis of the $|\mathscr{S}, \mathscr{M}\rangle$, its matrix is

\begin{tabular}{c|cccc}
$\hat{\mathcal{H}}_{S}$ & $|3 / 2\rangle$ & $|1 / 2\rangle$ & $|-1 / 2\rangle$ & $|-3 / 2\rangle$ \\
\hline$\langle 3 / 2|$ & $D+\frac{3}{2} \beta_{e} g B_{Z}$ & $\frac{\sqrt{3}}{2} \beta_{e} g\left(B_{X}-i B_{Y}\right)$ & $\sqrt{3} E$ & 0 \\
$\langle 1 / 2|$ & $\frac{\sqrt{3}}{2} \beta_{e} g\left(B_{X}+i B_{Y}\right)$ & $-D+\frac{1}{2} \beta_{e} g B_{Z}$ & $\beta_{e} g\left(B_{X}-i B_{Y}\right)$ & $\sqrt{3} E$ \\
$\langle-1 / 2|$ & $\sqrt{3} E$ & $\beta_{e} g\left(B_{X}+i B_{Y}\right)$ & $-D-\frac{1}{2} \beta_{e} g B_{Z}$ & $\frac{\sqrt{3}}{2} \beta_{e} g\left(B_{X}-i B_{Y}\right)$ \\
$\langle-3 / 2|$ & 0 & $\sqrt{3} E$ & $\frac{\sqrt{3}}{2} \beta_{e} g\left(B_{X}+i B_{Y}\right)$ & $D-\frac{3}{2} \beta_{e} g B_{Z}$ \\
& & &
\end{tabular}

The energies from diagonalization of this spin Hamiltonian are plotted as functions of $B$ for $g=2, D=-10 \mathrm{~cm}^{-1}$ and $E=1 \mathrm{~cm}^{-1}$ on Fig. 0.2. The two Kramers doublets $| \pm 1 / 2\rangle$ and $| \pm 3 / 2\rangle$ are split by an energy $2 \sqrt{D^{2}+3 E^{2}}$. With $D<0$, the $| \pm 3 / 2\rangle$ doublet is the lowest. When $E=0$, the $| \pm 3 / 2\rangle$ doublet is purely axial along $Z$ with a magnetization of $3 / 2 g$ while the $| \pm 1 / 2\rangle$ doublet has a magnetization $1 / 2 g$ along $Z$ and $g$ along $X$ and $Y$. The $E$ parameter induces some $| \pm 1 / 2\rangle$ component in the ground state in zero field and couples the two doublets through the Zeeman interaction. The magnetization becomes less axial. When $D$ is large, the second Kramers doublet may not be detected by EPR, even with HF-HF EPR. In this case, the ground Kramers doublet can be modeled with a restricted model space with $\mathscr{S}=1 / 2$; the spin Hamiltonian is then pure Zeeman and the g matrix is purely axial $g_{Z}=3 g$ and $g_{X}=g_{Y}=0$ for $D<0$ and $g_{Z}=g$ and $g_{X}=g_{Y}=2 g$ for $D>0$.

Most cases with large values of $\mathscr{S}$ are in the weak SO coupling limit with $\mathscr{S}$ close to the real spin $S$ of the spin-free state. When there is no very low lying state, the orbit contribution arises through second-order coupling with the excited states, the Zeeman interaction is almost isotropic and the anisotropic behavior of the magnetization arises from the $\mathbf{D}$ tensor. In this case, whatever the value of $\mathscr{S}$ is, the effective Hamiltonian technique permits a simple assignment between the 'real' wave functions to the pseudo-spin components. 

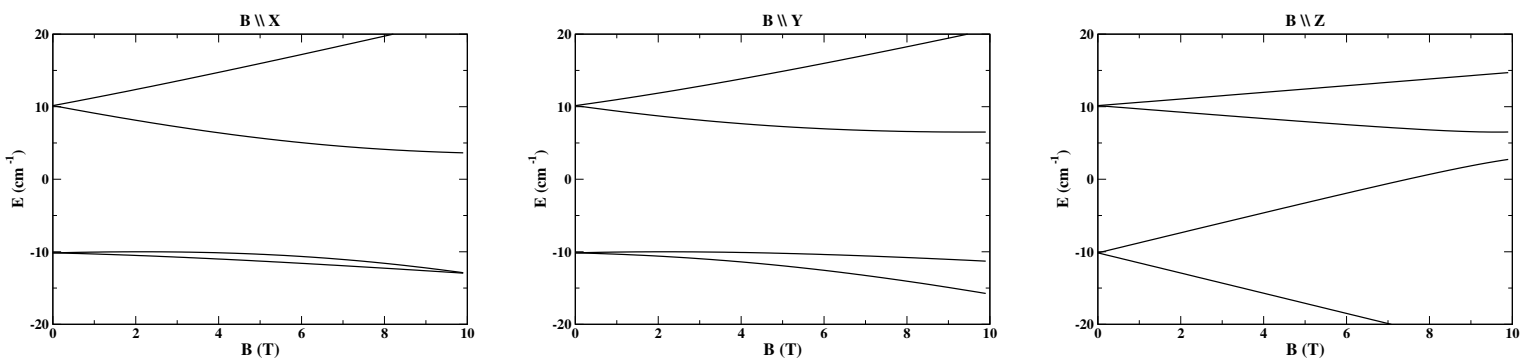

Fig. 0.2 Energy versus magnetic field for $\mathscr{S}=3 / 2$ as solution of Eq. 0.54 for $g=2, D=-10$ $\mathrm{cm}^{-1}$ and $E=1 \mathrm{~cm}^{-1}$

\subsection{Signs of EPR $g$-factors and hyperfine couplings}

In the Zeeman term of Eq. 0.6, the matrix g links the pseudo-spin operator $\hat{\mathscr{S}}$, which acts in the spin space, with the magnetic field, which acts in the physical space. Rotations in each of the two spaces are a-priori disconnected and, consequently, the matrix $\mathbf{g}$ is not a tensor. It is rather arbitrary since any rotation in the spin space affects the g matrix but gives the same electronic magnetic moment $\boldsymbol{\mu}$, which is the physical observable coupling to the magnetic field. The $g$-factors are calculated as the square roots of the principal values of the $\mathbf{G}$ tensor of Equation 0.15, which therefore determines the absolute values of the $g$-factors but does not provide any information about their sign. Experimentally, the $g$-factors are deduced from Equation 0.7. Conventional EPR does not provide the sign of the $g$-factors. In the weak SO limit, they are close to $g_{e} \simeq 2$ and they are positive.

Let $X, Y, Z$ denote the magnetic axes of a system. Pryce has shown [38] that the sign of the product $g_{X} g_{Y} g_{Z}$ determines the direction of the precession of the magnetic moment around the magnetic field. Experimentally, this sign has been measured for octahedral compounds where the three factors are identical. Negative signs were deduced relative to the sign of the hyperfine coupling [39] for $\mathrm{PaCl}_{6}^{2-}$ as well as [40] for $\mathrm{UF}_{6}^{-}$. The sign of $g_{X} g_{Y} g_{Z}$ was found to be negative as well for $\left[\mathrm{NpO}_{2}\left(\mathrm{NO}_{3}\right)_{3}\right]^{-}[41]$.

The sign of $g_{X} g_{Y} g_{Z}$ defines the sign of the Berry phase of a pseudo-spin applied in an applied magnetic field. [42] In the case of $\mathscr{S}=1 / 2$,

$$
\boldsymbol{\mu}^{X} \boldsymbol{\mu}^{Y} \boldsymbol{\mu}^{Z}=i g_{X} g_{Y} g_{Z} \mathbf{I}_{2}
$$

where $\boldsymbol{\mu}^{u}$ represents the electron magnetic moment $\hat{\mu}_{u}$ in the basis of the doublet state components, as defined in Section 0.2 .1 , and $\mathbf{I}_{2}$ is the $2 \times 2$ identity matrix. This product of matrices is invariant by rotation in the Hilbert spin space and therefore does not need any assignment between the two physical wave functions with the $|\mathscr{S}, \mathscr{M}\rangle$ pseudo-spin eigenfunctions. Eq. 0.55 is easily calculated $a b$-initio and gives access to the sign of the product of the three $g$-factors. 
In the case of symmetric molecules, the degree of arbitrariness of the matrix $\mathbf{g}$ can be reduced by imposing symmetry constraints on the pseudo-spin. More specifically, the pseudo-spin may be required to behave under spatial rotations $\hat{\theta}$ as a spin operator, up to a multiplicative function. [43] The two physical kets $|\lambda, 1\rangle$ and $|\lambda, 2\rangle$ span an irrep $\Gamma$ of the point group of the molecule, while the two components $|\alpha\rangle$ and $|\beta\rangle$ of a spin $S=1 / 2$ span an irrep $\Gamma_{S}$. If one can find a real scalar function $\phi$ such

$$
\begin{aligned}
& |\lambda, 1\rangle=\phi|\alpha\rangle \\
& |\lambda, 2\rangle=\hat{\theta}|\lambda, 1\rangle=\phi|\beta\rangle
\end{aligned}
$$

the two $|\lambda, 1\rangle$ and $|\lambda, 2\rangle$ are properly defined for the rotations of the molecule. This implies that $\Gamma=\Gamma_{\phi} \otimes \Gamma_{S}$ with $\Gamma_{\phi}$ being the irrep of $\phi$. Since the pseudo-spin and the multiplet are supposed to have the same degeneracy, $\Gamma_{\phi}$ must be a one-dimensional symmetry species. For example, in the case of the octahedral $\mathrm{AnX}_{6}^{\mathrm{q}-}$ complexes with $5 f^{1}$ configuration, the ground state is of symmetry $E_{5 / 2 u}$ and $\Gamma_{S}=E_{1 / 2 g}$. It follows that $\Gamma_{\phi}=A_{2 u}$ since $E_{5 / 2 u}=A_{2 u} \otimes E_{1 / 2 g}$ : the decomposition of Eq. (0.56) is uniquely defined and one can determine the signs of the $g$-factors. [44]. By symmetry, the three principal $g$-factors are equal and their sign is equal to the sign of the product.

The case of the neptunyl ion, $\mathrm{NpO}_{2}^{2+}$, is different. [45] The free ion is linear and has a non-zero principal $g_{\|}$-factor in the direction $\|$, parallel to the molecular axis. The components $g_{\perp}$ perpendicular to the axis are zero, however. This means that $g_{X} g_{Y} g_{Z}=0$, i.e. this product conveys no information about the sign of $g_{\|} \cdot \operatorname{In} \mathcal{D}_{\infty h}$, the ground state of neptunyl is $E_{5 / 2 u}$ and $\Gamma_{S}=E_{1 / 2 g}$. There is, however, no onedimensional irrep satisfying $E_{5 / 2 u}=\Gamma \otimes E_{1 / 2 g}$ and therefore the decomposition as in Eq. (0.56) is not possible. With equatorial ligands, the symmetry of the neptunyl is lowered, either to $\mathcal{D}_{3 h}$, e.g. in $\left[\mathrm{NpO}_{2}\left(\mathrm{NO}_{3}\right)_{3}\right]^{-}$, or to $\mathcal{D}_{4 h}$, e.g. in $\left[\mathrm{NpO}_{2} \mathrm{Cl}_{4}\right]^{2-}$. In both cases, the two equatorial $g_{\perp}$-factors are equal and $g_{\|}$has the sign of the product of the three $g$-factors. This sign is experimentally negative for the first complex. [41] According to Eq. 0.55, ab-initio calculations give a negative sign for the nitrate complex but a positive sign for the chloride. The ground state of $\left[\mathrm{NpO}_{2}\left(\mathrm{NO}_{3}\right)_{3}\right]^{-}$is of symmetry $E_{1 / 2}$, and in the $\mathcal{D}_{3 h}$ double group $\Gamma_{S}=E_{1 / 2}$. The scalar $\phi$ function belongs either to $A_{1}^{\prime}$ or to $A_{2}^{\prime}$ since $E_{1 / 2}=A_{1(2)}^{\prime} \otimes E_{1 / 2}$. The decomposition of Equation 0.56 leads in both cases to the same negative sign of $g_{\|}<0$, but one of the solutions gives $g_{\perp}>0$ while the other one gives $g_{\perp}<0$. In this case, the use of symmetry arguments does not produce a unique sign of $g_{\perp}$. In the same way, the ground state of $\left[\mathrm{NpO}_{2} \mathrm{Cl}_{4}\right]^{2-}$ is of symmetry $E_{3 / 2 u}, \Gamma_{S}=E_{1 / 2 g}$, and $\phi$ belongs to either $B_{1 u}$ or $B_{2 u}$. Both solutions give $g_{\|}>0$ but one gives $g_{\perp}>0$ while the other one gives $g_{\perp}<0$. Therefore, the individual signs of the $g$-factors of these neptunyl complexes can not be determined. It has been proposed [43] that the sign of $g$-factors in the case of an arbitrarily distorted complex could be determined by considering an adiabatic distortion of the complex towards a symmetric system for which the signs are well defined. But the analysis above shows that even in symmetrical cases, the signs of individual $g$-factors may not be unique.

While the sign of the product of the three $g$-factors can be related to an observable, namely the sense of the precession of the magnetic moment around the 
magnetic field, it appears that it is not possible in general to determine a specific sign of each individual $g$-factor, even in the case of molecules with high symmetry. Anyhow, the decomposition of Eq. 0.56 permits constructing a set of doublet components which behave as the components of a spin under the symmetry operations of a molecule.

\subsection{Selected case studies}

In this section, selected examples are presented where EPR parameters have been calculated with relativistic two-step complete active space (CAS) wavefunction methods (treating SO coupling by state interaction) and KS methods, drawing from the authors' research.

In the CAS approaches, first, the wave functions are calculated in the absence of magnetic fields and, in a second step, EPR parameters are deduced from the wave functions. In two-step approaches, the quality of the wave function depends on the quality of the basis set, on the size of the active space, on the introduction of the dynamical correlation, and on the number of states included in the state interaction for the calculation of the SO coupling. In all cases, for a metal in $n d(f)^{l}$ configuration, minimal active space includes the $l$ electrons in the 5(7) $n d(f)$ orbitals. Such a minimal active space is often sufficient for the description of $f$ elements as far as $g$-factors and ZFS is concerned. In order to get accurate HFC interactions, the active spaces must allow for spin polarization to take place. For transition metals, also for ZFS and $g$-factors the active spaces should be increased with some correlating orbitals, namely the double shell $n d^{\prime}$ orbitals and some orbitals of the ligands, namely the orbitals the most involved in the bonding with the metal ion. Except for lanthanides, the inclusion of the dynamical correlation with perturbation theory tends to improve the results. For the state interaction, all the states with the same spin as the ground term of the free ion arising from the $n d(f)^{l}$ configuration are usually included. The lowest states with $S \pm 1$ should often be included as well.

Once the wave functions are calculated, the model space must be chosen. In the case of Kramers doublets, the spin Hamiltonian comprises only the Zeeman term linear in $\mathscr{S}$ and the g-factors are calculated according to Equation 0.15. It is illustrated below with the example of neptunyl $\mathrm{NpO}_{2}^{2+}$. For non-Kramers doublets, a ZFS parameter must be added as shown below for the plutonyl $\mathrm{PuO}_{2}^{2+}$. The case $S=1$ is illustrated with a complex of $\mathrm{Ni}(\mathrm{II})$ where the pseudo-spin is very close to the real spin. Two examples are presented for $S=3 / 2$ : a complex of Co(II) with a ZFS splitting and a octahedral complex of $\mathrm{Np}(\mathrm{IV})$ without ZFS splitting but with a large third-order term in the pseudo-spin Hamiltonian. Finally, the $S=2$ case is illustrated with a high-spin complex of $\mathrm{Fe}(\mathrm{VI})$ where there is a low lying SF state. These calculations are all based on the second-order DKH operator with SO coupling treated by an atomic mean-field integral procedure. This section concludes with selected examples for hyperfine coupling extracted from KS calculations, where the relativistic effects are treated with the help of ZORA. 
The neptunyl ion $\mathrm{NpO}_{2}^{2+}$ is a linear complex of $\mathrm{Np}^{6+}$ in $5 f^{1}$ configuration. [45] The $5 f$ orbitals split due the interaction with the two oxygen atoms, the three orbitals of symmetry $\sigma$ and $\pi$ are antibonding and are destabilized. The remaining four orbitals, $\delta$ and $\phi$, are non bonding and are occupied with the single electron. The ground state is of symmetry $E_{5 / 2 u}$ of the $\mathcal{D}_{\infty h}$ group and is close to the $M_{J}= \pm 5 / 2$ components of the $J=5 / 2$ term of the free ion. Results are summarized in Table $0.1 g_{\|}= \pm 4.23$ is close to the $2 g_{J} J=30 / 7$ value of the free ion limit for a $|5 / 2, \pm 5 / 2\rangle$ doublet. The spin and orbital contributions to the $g$-factors are determined by turning off the orbital and spin term respectively in the Zeeman interaction. The orbital contribution is the largest and opposite to the spin one, as it is the case for the free ion where spin and orbit are in opposite direction since the open shell is less than half filled and $L>S$.

The $g$-factors of the ground state of $\mathrm{NpO}_{2}^{2+}$ were measured by EPR spectroscopy in the solid state diluted either in $\mathrm{CsUO}_{2}\left(\mathrm{NO}_{3}\right)_{3}$ or in $\mathrm{Cs}_{2} \mathrm{UO}_{2} \mathrm{Cl}_{4}$. The $g$-factors of the excited states were deduced from the absorption bands in a magnetic field. In the first environment, three nitrate ligands are in the equatorial plane of $\mathrm{NpO}_{2}$ leading to a local $\mathcal{D}_{3 h}$ symmetry while in the latter, there are four chloride with a local $\mathcal{D}_{4 h}$ symmetry. In the first complex, the $\phi$ orbitals split by interaction with the orbitals of the equatorial ligands and their orbital moment is partially quenched. It gives rise to a magnetic moment in the equatorial direction (see Table 0.1) dominated by the spin contribution; in this case, spin and orbit are opposite. In $\mathcal{D}_{4 h}$, the $\delta$ orbitals split by mixing with the orbitals of the ligands quenching their orbital moment. One obtains again a magnetic moment in the equatorial plane almost as large as the axial one. It should be noticed that in this case, the spin and orbital contributions of the axial component have the same sign, and are therefore additive. The main effect of the environment is to affect the ratio of $\delta$ and $\phi$ orbitals in the ground state and it is this ratio which determines the magnetic properties. The effect of the ligands is so large in the chloride environment that there is no relationship anymore with the properties of the free actinyl ion.

As pointed out already, even-electron systems may have doubly degenerate states in presence of rotational symmetry, or almost degenerate states with a small energy gap. In this case, the pseudo-spin is $\mathscr{S}=1 / 2$. But, while in the spin space the kets behave as $\hat{\Theta}|1 / 2, \pm 1 / 2\rangle= \pm|1 / 2, \mp 1 / 2\rangle$ under time reversal, with $\hat{\Theta}$ being the time-reversal operator, in the real space $\hat{\Theta}|\lambda, a\rangle=|\lambda, a\rangle$ since there is an even number of electrons. The states $|\lambda, a\rangle$ have no magnetic moment but the magnetic

Table 0.1 Calculated and experimental g-factors for $\mathrm{NpO}_{2}^{2+}$ and $\mathrm{PuO}_{2}^{2+} \cdot g^{S}$ and $g^{L}$ are the spin and orbital contributions to the calculated $g$-factors. Data taken from References 45, 46 .

\begin{tabular}{|c|c|c|c|c|c|c|c|c|c|c|}
\hline & \multicolumn{2}{|c|}{$\mathrm{NpO}_{2}^{2+}$} & \multicolumn{2}{|c|}{$\left[\mathrm{NpO}_{2}\left(\mathrm{NO}_{3}\right)_{3}\right]^{-}$} & \multicolumn{2}{|c|}{$\left[\mathrm{NpO}_{2} \mathrm{Cl}_{4}\right]^{2-}$} & \multicolumn{2}{|c|}{$\mathrm{PuO}_{2}^{2+}$} & \multicolumn{2}{|c|}{$\left[\mathrm{PuO}_{2}\left(\mathrm{NO}_{3}\right)_{3}\right]$} \\
\hline & & $\perp$ & $\|$ & $\perp$ & $\|$ & $\perp$ & $\|$ & $\perp$ & & $\perp$ \\
\hline & \pm 4.24 & 0.00 & -3.49 & \pm 0.23 & 1.76 & \pm 1.50 & 6.09 & 0.00 & 5.92 & 0.00 \\
\hline$g^{L}$ & \pm 5.76 & 0.00 & -4.69 & $\mp 0.63$ & 1.72 & $\mp 2.42$ & 9.90 & 0.00 & 9.62 & 0.00 \\
\hline$g^{S}$ & $\mp 1.52$ & 0.00 & 1.20 & \pm 0.86 & 0.04 & $\pm 0.92 \mathrm{i}$ & -3.80 & 0.00 & -3.68 & 0.00 \\
\hline $\exp$ & & & $3.36 ; 3.405$ & $0.20 ; 0.205$ & $1.32 ; 1.38$ & 1.30 & & & 5.32 & 0.00 \\
\hline
\end{tabular}


moment arises from the coupling between the two states $\langle\lambda, a|\hat{\boldsymbol{\mu}}| \lambda, b\rangle \neq 0$ if $a \neq b$. It follows that the spin Hamiltonian takes the following form

$$
\hat{\mathcal{H}}_{S}=g_{\|} \beta_{e} \hat{\mathscr{S}}_{z} B_{z}+\Delta \hat{\mathscr{S}}_{x}
$$

assigning the $|1 / 2, \pm 1 / 2\rangle \equiv \frac{1}{\sqrt{2}}(|\lambda, a\rangle \pm i|\lambda, b\rangle)$. The second term of Equation 0.57 is a ZFS term linear in $\mathscr{S}$ and is not even with time reversal. In this equation, $x$ and $y$ do not refer to spatial directions and the Hamiltonian is not invariant under rotations. $g_{\|}$is a number. Although ill defined for symmetries, this Hamiltonian is used to fit EPR spectra of non Kramers systems and its parameters can be determined from $a b$-initio calculation.

The plutonyl ion $\mathrm{PuO}_{2}^{2+}$ is the analog of $\mathrm{NpO}_{2}^{2+}$ with a $5 f^{2}$ configuration. The ground state is of $4_{g}$ symmetry, close to the $M_{J}= \pm 4$ of the ground free ion ${ }^{3} H_{4}$ term. The calculated $g$-factor (see Table 0.1 ) is close to $2 g_{J} J=6.4$ of the free ion. The equatorial component is zero and the spin and orbital contributions to the axial component have opposite signs. The EPR spectrum of plutonyl has been measured diluted in a diamagnetic crystal, forming $\left[\mathrm{PuO}_{2}\left(\mathrm{NO}_{3}\right)_{3}\right]^{-}$clusters. The equatorial moment remains zero, even with the lowering of the symmetry since there is an even number of electrons in the molecule. In this case, the splitting of the $\phi$ orbitals has less effect than in neptunyl since the configuration of the ground state remains mostly $\phi^{1} \delta^{1}$.

In the complex $\left[\mathrm{Ni}(\mathrm{II})\left(\mathrm{HIM}_{2}-\mathrm{py}\right)_{2}\left(\mathrm{NO}_{3}\right)\right]^{+}$, the $\mathrm{Ni}(\mathrm{II})$ ion $\left(3 d^{8}\right.$ configuration $)$ is in a pseudo-octahedral environment. Magnetization measurements, HF-HF-EPR studies, and frequency domain magnetic resonance spectroscopy (FDMRS) studies indicated the presence of a very large Ising-type anisotropy with an axial ZFS parameter $D=-10.1 \pm 0.1 \mathrm{~cm}^{-1}$ and a rhombic ZFS parameter of $E=0.3 \pm 0.1 \mathrm{~cm}^{-1}$ [47]. The spectrum is fitted with an isotropic $g_{i s o}=2.17$. In an octahedral ligand field, the SR ground state is a ${ }^{3} A_{2 g}$ with a $t_{2 g}^{6} e_{g}^{2}$ configuration. This state is orbitally non-degenerate and triply degenerate for the spin. With SO coupling, it becomes a $T_{2}$ state and remains triply degenerate. A distortion of the octahedral environment removes this degeneracy, creating three non-degenerate states. SO coupling is dominated by coupling to a ${ }^{3} T_{2 g}$ state with configuration $t_{2 g}^{5} e_{g}^{3}$. The latter is triply degenerate in octahedral symmetry and also splits in three states when the symmetry is lowered. The model space consists in the three states arising from the ground ${ }^{3} A_{2 g}$ state and $\mathscr{S}=1$.

According to SO-CASPT2 calculations, the ${ }^{3} T_{2 g}$ state splits in three components at 7750, 10088 and $10504 \mathrm{~cm}^{-1}$ above the energy of the ground state. This large splitting is at the origin of the large ZFS in this molecule. According to Equation 0.51 , one gets $D=-11.53 \mathrm{~cm}^{-1}$ and $E=0.48 \mathrm{~cm}^{-1}$. The directions of the principal axes of the $\mathbf{d}$ tensor are depicted on Fig. 0.3. The norms of the projections of the model wave functions in the target space $\mathcal{P}|\lambda, a\rangle$ are 0.99 and their overlap about $10^{-4}$. It follows that the matrix $\mathbf{C}$ is close to being orthogonal, and close to the rotation matrix $\mathcal{R}$ of Equation 0.49. Equations 0.49 and 0.51 provide similar results. 


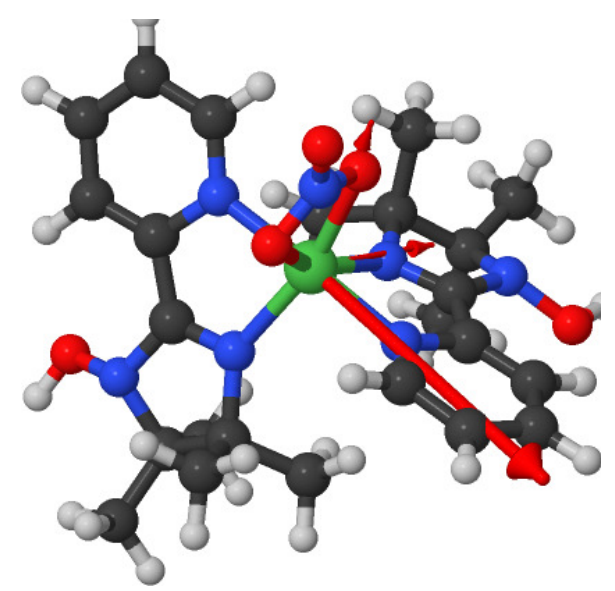

Fig. 0.3 The $\left[\mathrm{Ni}(\mathrm{II})\left(\mathrm{HIM}_{2}-\mathrm{py}\right)_{2}\left(\mathrm{NO}_{3}\right)\right]^{+}$molecule. The thick arrows shows the direction of the axial magnetization and the thiner arrows the two other principal axes of the $\mathbf{d}$ tensor.

The $g$-factors determined by the projection procedure, Equation (0.45), and the matching procedure are the same $g_{X}=2.21, g_{Y}=2.22$ and $g_{Z}=2.30$. The principal axes of the $\mathrm{g}$ tensor are very close to those of the $\mathbf{d}$ tensor even if the molecule has no symmetry. The $g$-factors are almost equal and the Zeeman interaction is almost isotropic. This means that the magnetic behavior is dictated by the principal axes of the ZFS tensor $\mathbf{d}$. The spin contribution to the $g$-factors is $g_{X}^{S}=g_{Y}^{S}=1.998, g_{Z}^{S}=1.992$ and the orbital contributions are $g_{X}^{L}=0.225$ $g_{Y}^{L}=0.222$ and $g_{Z}^{L}=0.306$. As expected for a weak SO case, the spin contribution is by far the largest. But it is isotropic and close to 2 . The orbit contribution is smaller, but it brings the departure from the free electron and the anisotropy of the Zeeman interaction.

In $\mathrm{HgCo}(\mathrm{NCS})_{4}$, the $\mathrm{Co}$ (II) ion has a $3 d^{7}$ configuration and is in a pseudotetrahedral environment. The $\left[\mathrm{Co}(\mathrm{NCS})_{4}\right]^{2-}$ molecule belongs to the $S_{4}$ point group. In $T_{d}$, the ground state would be a four-fold degenerate ${ }^{4} A_{2}$, but in the lower symmetry, this state splits in two Kramers doublets. The spin Hamiltonian parameters were deduced from susceptibility data measured with a SQUID in the range 1.7-300 $\mathrm{K}$ [48]; $D$ lies between 10 and $11 \mathrm{~cm}^{-} 1, g_{\|}=2.168$, and $g_{\perp}=2.251$.

The splitting between the two Kramers doublets calculated with SO-CASPT2 is $16.2 \mathrm{~cm}^{-1}$. Due to the symmetry of the molecule, all spin Hamiltonian tensors have the same principal axis and the spin Hamiltonian takes the following form

$$
\begin{aligned}
\hat{\mathcal{H}}_{S} & =\beta_{e}\left(g_{\perp} B_{X} \hat{\mathscr{S}}_{X}+g_{\perp} B_{Y} \hat{\mathscr{S}}_{Y}+g_{\|} B_{Z} \hat{\mathscr{S}}_{Z}\right) \\
& +\beta_{e}\left(g_{\perp}^{\prime} B_{X} \hat{\mathscr{S}}_{X}^{3}+g_{\perp}^{\prime} B_{Y} \hat{\mathscr{S}}_{Y}^{3}+g_{\|}^{\prime} B_{Z} \hat{\mathscr{S}}_{Z}^{3}\right) \\
& +D\left(\hat{\mathscr{S}}_{Z}^{2}-\frac{5}{4}\right)+E\left(\hat{\mathscr{S}}_{X}^{2}-\hat{\mathscr{S}}_{Y}^{2}\right)
\end{aligned}
$$


The matrix $\mathbf{g}^{\prime}$ is reduced to its diagonal elements. The ZFS tensor $\mathbf{d}$ is calculated by the effective Hamiltonian technique ; the $Z$ axis lies along the $\mathcal{C}_{2}$ axis, and $D=$ $8.1 \mathrm{~cm}^{-1}$ and $E=0$. The validity of the effective Hamiltonian procedure can be assessed by the projection of the $|\lambda, a\rangle$ on the $\left|\lambda^{\prime}, M\right\rangle$, more than 0.99 and the overlap between the projected vectors, less than $10^{-4}$. The projection technique provides the tensor $\mathcal{A}$ defined in Eq. 0.45 leading to $g_{\|}=2.173$ and $g_{\perp}=2.255$. The matching technique permits the determination of both linear and cubic terms : one gets $g_{\|}=2.178, g_{\|}^{\prime}=-0.002, g_{\perp}=2.263, g_{\perp}^{\prime}=-0.004$. The Zeeman interaction is mostly isotropic and the cubic term is negligible. The two techniques give close but not similar $g$-factors due to the neglect of the cubic term in the first one. These values are in very good agreement with the experimental ones. The spin (orbital) contributions are for $g_{\perp} 1.992(0.271)$, for $g_{\perp}^{\prime}-0.0002(-0.004)$, for $g_{\|}$ $1.989(0.189)$ and for $g_{\|}^{\prime}-0.0002(-0.002)$. The spin contribution is again the largest, isotropic and close to 2 . The orbital one is more anisotropic and provides the only contribution to the cubic term. As it was the case for the Ni(II) complex, in the weak SO limit, the anisotropy of the magnetic property is determined by the ZFS interaction.

Another description is to consider the two Kramers doublets independently, each being described by a $\mathscr{S}=1 / 2$. One gets for the first Kramers doublet $g_{1 \|}=2.18$ and $g_{1 \perp}=4.50$ and for the second one $g_{2 \|}=6.52$ and $g_{2 \perp}=0.1$. This description does not give information on the second order Zeeman interaction between the two Kramers doublets. But in the case of a large ZFS, the transition between the two Kramers doublets can not be induced even using HFHF EPR and one only determines the magnetic properties of the ground Kramers doublet. In this scheme, the magnetic anisotropy is described by the Zeeman interaction.

Another example for $\mathscr{S}=3 / 2$ is the $\mathrm{NpCl}_{6}^{2-}$ cluster diluted in $\mathrm{Cs}_{2} \mathrm{ZrCl}_{6}$. The $\mathrm{Np}^{4+}$ ion has a $5 f^{3}$ configuration and is in an octahedral environment. The ground state is a quartet $F_{3 / 2 u}$ (using Mulliken's notation, or $\Gamma_{8}$ in Bethe's notation) [49]. In the principal axis system, the spin Hamiltonian takes the form

$$
\hat{\mathcal{H}}_{S}=\beta_{e} g \hat{\mathscr{S}} \cdot \boldsymbol{B}+\beta_{e} g^{\prime}\left(B_{X} \hat{\mathscr{S}}_{X}^{3}+B_{Y} \hat{\mathscr{S}}_{Y}^{3}+B_{Z} \hat{\mathscr{S}}_{Z}^{3}\right)
$$

Due to the cubic symmetry, $\hat{\mathcal{H}}_{(1)}^{Z}$ is isotropic, $\hat{\mathcal{H}}_{(3)}^{Z}$ is invariant by 'changing' the axis by permutation $X \longleftrightarrow Y \longleftrightarrow Z$ but is not isotropic. There are two degrees of freedom, the two scalar $g$ and $g^{\prime}$ which were deduced from EPR measurements as $g=-0.516$ and $g^{\prime}=0.882$. These two numbers define the magnetization of the two pairs of Kramers doublets, $\left\langle \pm 3 / 2\left|\mu^{u}\right| \pm 3 / 2\right\rangle=\mp\left(\frac{3}{2} g+\frac{27}{8} g^{\prime}\right)$ and $\left\langle \pm 1 / 2\left|\mu^{u}\right| \pm 1 / 2\right\rangle=\mp\left(\frac{1}{2} g+\frac{1}{8} g^{\prime}\right)$. The matching technique permits to find the rotation $\mathcal{R}$ in the model space which assign the $|\lambda, a\rangle$ to the $|\mathscr{S}, \mathscr{M}\rangle$. One can find two possible assignments as was already pointed out by Bleaney, corresponding to the permutation $\left\{\Psi_{3 / 2} \leftrightarrow \Psi_{-1 / 2} ; \Psi_{-3 / 2} \leftrightarrow \Psi_{1 / 2}\right\}$. One of the solutions transforms under rotations as a spin $S=3 / 2$ while the other one does not. The solution given above fitting the EPR spectrum does not. From calculations one gets $g=-0.406$ and $g^{\prime}=0.785$. The spin and orbital contributions are 0.027 and -0.460 for $g$ and -0.250 and 1.285 for $g^{\prime}$. The accordance with the experimental values is reasonable. 
The spin and orbital contributions are opposite, the orbital one being the largest as in the free ion. The cubic term is by far non negligible, it is larger than the linear one, and opposite in sign. Due to the high symmetry of the complex, there is no ZFS. However, the cubic term plays an essential role for the Zeeman interaction.

When the complex is distorted, one gets two Kramers doublets with different energies. The two $g$-tensors are not collinear. Two pseudo-spins models can be used: Either one considers two distinct $\mathscr{S}=1 / 2$ each with its own $g$-tensor, and the two $g$-tensors are not collinear. The two spin spaces are coupled through 2 nd order Zeeman interaction. It is usually the way one describes the low-energy spectra of lanthanide ions. Another way is to describe the model space as an $\mathscr{S}=3 / 2$ case. The $\mathbf{g}$ and $\mathbf{g}^{\prime}$ tensor are not collinear in general, and even if this space is more complete that the previous description, the parameters are less intuitive.

In the $[\mathrm{FeLCl}]$ complex with $\mathrm{L}=\beta$-diketiminate, the $\mathrm{Fe}(\mathrm{II})$ is in $3 d^{6}$ high-spin configuration with a $S=2$ ground state. EPR measurements in the $\mathrm{X}$ band gives a quasidegenerate $M_{S}= \pm 2$ ground doublet with an axial $g=10.9$ and a small splitting of $\Delta=0.35 \mathrm{~cm}^{-1}$. The next component of the spin quintet are estimated to be higher than $150 \mathrm{~cm}^{-1}$ [50]. In a pure spin quintet, the $M_{S}= \pm 2$ components have a $g$-factor of 6 . The experimental value shows that there is a large orbital contribution due a partially quenching of the orbital moment and a low lying state. The CASPT2 calculation shows that without SO coupling the first excited state is another spin quintet lying $516 \mathrm{~cm}^{-1}$ above the ground state. With the SO coupling, the spin quintet becomes a ground doublet split by $0.34 \mathrm{~cm}^{-1}$ and the other components are 118, 132 and $180 \mathrm{~cm}^{-1}$ above. The $g$-factors of the ground doublet modelled with a $\mathscr{S}=1 / 2$ are given in Table 0.2 . The magnetization is along the $\mathrm{Fe}-\mathrm{Cl}$ bond with $g=10.9$ in perfect accordance with the experimental value. The spin contribution of 7.99 is the expected value for a spin quintet and one notices a large orbital contribution due to the low lying quintet state. The five components issued from the spin quintet may be modelled with a $\mathscr{S}=2$ pseudo-spin. In the case where all the matrices are collinear, the spin Hamiltonian is the following

$$
\begin{aligned}
\hat{\mathcal{H}}_{S} & =\beta_{e}\left(g_{X} B_{X} \hat{\mathscr{S}}_{X}+g_{Y} B_{Y} \hat{\mathscr{S}}_{Y}+g_{Z} B_{Z} \hat{\mathscr{S}}_{Z}\right) \\
& +\beta_{e}\left(g_{X}^{\prime} B_{X} \hat{\mathscr{S}}_{X}^{3}+g_{Y}^{\prime} B_{Y} \hat{\mathscr{S}}_{Y}^{3}+g_{Z}^{\prime} B_{Z} \hat{\mathscr{S}}_{Z}^{3}\right) \\
& +D\left(\hat{\mathscr{S}}_{Z}^{2}-2\right)+E\left(\hat{\mathscr{S}}_{X}^{2}-\hat{\mathscr{S}}_{Y}^{2}\right)+D^{\prime}\left(\hat{\mathscr{S}}_{Z}^{4}-\frac{34}{5}\right)+E^{\prime}\left(\hat{\mathscr{S}}_{X}^{4}-\hat{\mathscr{S}}_{Y}^{4}\right)
\end{aligned}
$$

The matrices $\mathbf{g}^{\prime}$ and $\mathbf{d}^{\prime}$ are reduced to their diagonal elements. All the spin parameters have been determined. $Z$ is along the $\mathrm{Fe}-\mathrm{Cl}$ axis. The $g$-factors and their spin and orbital contributions are summarized in Table 0.2. For the ZFS tensors, one finds $D=-58.0 \mathrm{~cm}^{-1}, E=2.0 \mathrm{~cm}^{-1}, D^{\prime}=3.3 \mathrm{~cm}^{-1}$ and $E^{\prime}=0.1 \mathrm{~cm}^{-1}$. The tensor of second order is largely dominant. While the rhombic term $E$ models the splitting between the two $M_{S}= \pm 2$ components, the parameter $D^{\prime}$ of the term of fourth order is necessary to model the whole spectrum of energy of the five pseudo-spin 
Table $0.2 g$-factors of FeLCl calculated with two model spaces $\mathscr{S}=1 / 2$ and $\mathscr{S}=2 \cdot g^{S}$ and $g^{L}$ are the spin and orbital contributions.

\begin{tabular}{|c|c|c|c|c|c|c|c|c|c|}
\hline & \multirow{2}{*}{\multicolumn{3}{|c|}{$\begin{array}{c}\mathscr{S}=1 / 2 \\
\mathrm{~g}\end{array}$}} & \multicolumn{6}{|c|}{$\mathscr{S}=3 / 2$} \\
\hline & & & & \multicolumn{3}{|c|}{$\mathrm{g}$} & \multicolumn{3}{|c|}{$\mathrm{g}^{\prime}$} \\
\hline & X & $\mathrm{y}$ & $\mathrm{Z}$ & X & $y$ & Z & X & $y$ & Z \\
\hline calc $g$ & 0.0 & 0.0 & 10.903 & 2.104 & 1.983 & 3.108 & -0.0154 & -0.013 & -0.094 \\
\hline$g^{S}$ & 0.0 & 0.0 & 7.994 & 2.000 & 1.965 & 2.000 & -0.016 & -0.012 & 0.000 \\
\hline$g^{L}$ & 0.0 & 0.0 & 2.909 & 0.104 & 0.018 & 1.108 & 0.002 & -0.001 & -0.094 \\
\hline $\exp$ & 0.0 & 0.0 & 10.9 & & & & & & \\
\hline
\end{tabular}

Table 0.3 Isotropic ${ }^{199} \mathrm{Hg}$ HFC constants, in units of MHz, calculated with different relativistic methods. ${ }^{a}$

\begin{tabular}{lrr}
\hline & \multicolumn{1}{c}{$\mathrm{HgF}$ HgAg } \\
\hline NR PN & $9173 \quad 2068$ \\
ZORA PN & 219583404 \\
ZORA FN & 191713094 \\
4-component PN 189273690 \\
4-component FN 168953285 \\
Expt. & $22163 \quad 2720$
\end{tabular}

${ }^{a} \mathrm{PN}=$ point nucleus, $\mathrm{FN}=$ finite nucleus (spherical Gaussian model), ZORA = zeroth-order regular approximation 2-component method. For citations of the original data see Table 3 of Reference 51.

components. It should be noticed that, due to the large coupling with the excited spin quintet, the projection of the five wave function in the target space is reduced to 0.89 and 0.95 . For the Zeeman interaction, as in $\mathrm{Co}(\mathrm{NCS})_{4}$, the linear term is largely dominant and the cubic one is negligible. The spin contribution is isotropic and close to 2 while the orbital contribution is very important in the $Z$ direction, larger than 1, and negligible in the two other ones.

Electronic EPR parameters can be calculated in many different cases. In transition metal complexes, the pseudo-spin is usually the spin of the ground state. In the case of a large ZFS, the model space can be reduced to the lower components and effective values of $g$-factors are measured and calculated. When the pseudo-spin is equal to the spin, the spin contribution to the $g$-factor is isotropic and close to 2 . The anisotropy of $\mathbf{g}$ arises from orbital contributions. For large values of the pseudospin, the higher order terms, in $\mathscr{S}^{3}$ for the Zeeman interaction and in $\mathscr{S}^{4}$ the ZFS interaction are much smaller than the $\mathscr{S}$ and $\mathscr{S}^{2}$ terms. Except in the case of a low lying excited state, the anisotropy of the magnetic properties arises from the ZFS tensor, the $g$-factors being almost isotropic.

In the case of complexes with heavy elements, the pseudo-spin is rarely larger than 2. Usually, the spin and orbital components are in opposite directions for less than filled open shells and in the same direction otherwise, as a reminiscence of the free ion. But in the case of the quenching of some orbitals due to the interaction with the ligands, this rule can be skewed for actinides. 


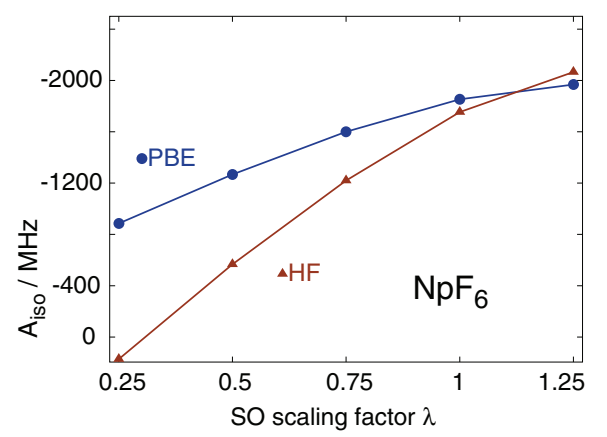

Fig. 0.4 ${ }^{237} \mathrm{~Np}$ HFC constant of $\mathrm{NpF}_{6}$ versus scaling of SO Hamiltonian integrals in the ground state calculation $(\lambda=0$ means SR, $\lambda=1$ means full SO). HF and DFT calculations (PBE functional) of Ref. 30 utilizing Eq. (0.22).

Regarding HFC, there are few examples highlighting the role of relativistic effects and finite nuclear volume effects better than ${ }^{199} \mathrm{Hg}$. Table 0.3 lists a collection of calculated (DFT, with a hybrid functional for NR and ZORA and a non-hybrid functional for the 4-component calculations) and experimental data for the radicals $\mathrm{HgH}$ and $\mathrm{HgF}$. The increase of the Hg HFC from NR to relativistic calculations, in particular for $\mathrm{HgH}$, is rather spectacular. The effect is mainly due to SR effects from the relativistic increase of the spin density at the $\mathrm{Hg}$ nucleus. The effects from a finite nuclear volume are also very large, roughly on the order of $10 \%$ relative to the total, and decrease the HFC. SO effects on mercury HFC constants tend to be relatively minor because it is dominated by contributions from the $\mathrm{Hg} 6 s$ orbital.

The situation is different for the $5 f^{1}$ complex ${ }^{237} \mathrm{~Np}$ and ${ }^{19} \mathrm{~F}$ HFC constants of $\mathrm{NpF}_{6}$. Experimentally, the isotropic $\mathrm{HFC}$ constants were found to be $-1995 \mathrm{MHz}$ for ${ }^{237} \mathrm{~Np}$ and $-73 \mathrm{MHz}$ for ${ }^{19} \mathrm{~F}$. For Np, the HFC tensor is isotropic due to the octahedral symmetry of the complex. For ${ }^{19} \mathrm{~F}$, there are two unique tensor components, $a_{\|}$in the direction of the $\mathrm{Np}-\mathrm{F}$ axes and a degenerate pair of $a_{\perp}$ perpendicular to the $\mathrm{Np}-\mathrm{F}$ axes, with $a_{\|}=-132, a_{\perp}=-42 \mathrm{MHz}$ experimentally. The unpaired electron is described at the SR level by a non-bonding Np $5 f$ orbital of $\delta$ symmetry with respect to the Np-F axes. Without SO coupling, the isotropic Np HFC is calculated to be much too small in magnitude, by a factor of 3 to 5 depending on the computational method. Spin polarization is responsible for the residual Np HFC and most of the fluorine HFC at the SR level. Under the SO interaction, there is a very dominant contribution to the Np HFC from the PSO mechanism. This mechanism can be interpreted as follows: Via SO coupling, the unpaired spin at Np creates a paramagnetic orbital current density in the $5 f$ shell which then interacts magnetically with the nuclear spin. The NMR shielding tensors for diamagnetic molecules afford a similar mechanism, except that a paramagnetic orbital current density is induced by the external magnetic field rather than via an unpaired spin and SO coupling.

Figure 0.4 shows the ${ }^{237} \mathrm{~Np}$ HFC constant for $\mathrm{NpF}_{6}$ calculated with HF theory and DFT using Equation (0.22). The horizontal axis indicates a parameter $\lambda$ used to scale the SO Hamiltonian integrals in the ground state calculations, meaning that $\lambda=0$ 
gives the SR limit. The fact that the data exhibit significant curvatures show that SO effects beyond first order are important and reduce the HFC magnitude. Indeed, calculations where $\mathrm{SO}$ coupling is treated with a linear-response method (to produce the PSO mechanism) over-estimate the Np HFC by $15 \%$ or more, depending on the functional used for the calculations. Further details and citations of prior studies where such SO scaling has been used can be found in Reference 30 .

For ${ }^{19} \mathrm{~F}$, CASSCF calculations recently indicated [52] that without SO coupling the HFC tensor anisotropy is not correctly predicted. With SO coupling, the calculations predict $a_{\|}$to be twice as large in magnitude as $a_{\perp}$, with the latter close to experiment. The magnitude of the calculated $a_{\|}$appears to be underestimated. However, the measurements were performed on a solid, with $\mathrm{NpF}_{6}$ diluted in a $\mathrm{UF}_{6}$ host crystal, while the calculations were for an isolated molecule. It is presently unknown precisely how solid state packing affects the fluorine HFC tensors.

The CAS calculations of $g$-tensors and ZFS discussed above were performed with the MOLCAS suite of programs. For the benefit of the reader, details of the computations are given here. $\mathrm{NpO}_{2}^{2+},\left[\mathrm{NpO}_{2}\left(\mathrm{NO}_{3}\right)_{3}\right]^{-},\left[\mathrm{NpO}_{2} \mathbf{C l}_{4}\right]^{2-}, \mathbf{P u O}_{2}^{2+}$ and $\left[\mathrm{PuO}_{2}\left(\mathrm{NO}_{3}\right)_{3}\right]^{-}$: Results are given with ano.rcc basis sets of TZP quality at the SOCASPT2 level with CAS $(7,10)$ comprising the $75 f$ orbitals and the two bonding $\sigma$ and $\pi$ orbitals. The environment is described by ECP and point charges. The state interaction for the calculation of the SO coupling is performed with 6 spin doublets. $\left[\mathbf{N i}(\mathbf{I I})\left(\mathbf{H I M}_{2}-\mathbf{p y}\right)_{\mathbf{2}}\left(\mathbf{N O}_{3}\right)\right]^{+}$: Results are given with ano.rcc basis sets of TZP quality on the Ni and DZP on the ligands at the SO-CASPT2 level with CAS $(8,10)$ comprising the $83 d$ electrons within a double shell of $d$ orbitals. The state interaction for the calculation of SO coupling is performed with 10 triplets and 15 singlets. Similar calculations are published in reference [53]. $\mathbf{H g C o}(\mathrm{NCS})_{4}$ : Calculations are performed with ano.rcc basis sets of TZP quality for the $\left[\mathrm{Co}(\mathrm{NCS})_{4}\right]^{2-}$ complex. The complex is embedded in point charges describing the rest of the crystal. $\mathrm{Hg}^{2+}$ ions are described by ECPs and other ions by point charges within a radius of $10 \AA$. The cluster is described at the SO-CASPT2 level with 10 spin quartets

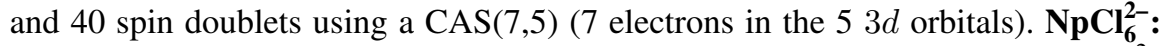
Calculations are performed with ano.rcc basis sets of TZP quality for the $\left[\mathrm{NpCl}_{6}\right]^{2-}$ complex. The complex is embedded in point charges and model potentials describing the rest of the crystal. $\mathrm{Hg}^{2+}$ ions are described by ECPs and other ions by point charges. The cluster is described at the SO-CASPT2 level with 35 quartets and 84 doublets using a CAS $(3,7)$ ( 3 electrons in the $75 f$ orbitals). [ $\mathbf{L F e C l}$ ]: Results are given with ano.rcc basis sets of TZP quality on the Fe and DZP on the ligands at the SO-CASPT2 level with CAS(6,5) comprising the $63 d$ electrons within the $53 d$ orbitals. The state interaction for the calculation of $\mathrm{SO}$ coupling is performed with 5 quintets, 15 triplets and 10 singlets. 


\subsection{Summary}

There is a large variety of techniques available for $a b$-initio calculations of EPR parameters. Nonetheless, it can be a complicated task to extract EPR spin Hamiltonian parameters from ab-initio calculations if SO coupling is strong, if the degeneracy of the state of interest is higher than two-fold, or for non-Kramers doublets. In the limit of weak SO coupling, the effective Hamiltonian technique is well established. For strong SO coupling, the wavefunctions should include SO coupling variationally or via state interaction. KS methods can be an effective alternative to wavefunction based methods.

Acknowledgements J.A. acknowledges support of his research on EPR and NMR parameters of open-shell heavy metal complexes by the U.S. Department of Energy, Office of Basic Energy Sciences, Heavy Element Chemistry program, under grant DE-FG02-09ER16066.

Key words: Electron paramagnetism, electron paramagnetic resonance, spin Hamiltonian, spin orbit coupling, hyperfine coupling, electron $g$-factor, Zeeman interaction, electron spin, nuclear spin, finite nucleus effects

\section{References}

1. N.M. Atherton, Principles of Electron Spin Resonance. Ellis Horwood series in physical chemistry (Prentice Hall, New York, 1993)

2. P.H. Rieger, Electron spin resonance. Analysis and interpretation (The Royal Society of Chemistry, Cambridge, UK, 2007)

3. J.E. Harriman, Theoretical foundations of electron spin resonance (Academic Press, New York, 1978)

4. A. Abragam, B. Bleaney, Electron paramagnetic resonance of transition ions (Clarendon Press, Oxford, 1970)

5. P.J. Mohr, B.N. Taylor, D.B. Newell, Rev. Mod. Phys. 84, 1527 (2012)

6. J.E. Butler, C.A. Hutchison, Jr., J. Chem. Phys. 74(6), 3102 (1981)

7. J.S. Griffith, Mol. Phys. 12, 359 (1967)

8. F. Neese, E.I. Solomon, Inorg. Chem. 37, 6568 (1998)

9. H. Bolvin, ChemPhysChem 7, 1575 (2006)

10. L.F. Chibotaru, L. Ungur, J. Chem. Phys. 137, 064112 (2012)

11. L.F. Chibotaru, Adv. Chem. Phys. 153, 397 (2013)

12. B.O. Roos, P.R. Taylor, P.E.M. Siegbahn, Chem. Phys. 48, 157 (1980)

13. K. Andersson, P.A. Malmqvist, B.O. Roos, A.J. Sadlej, K. Wolinski, J. Phys. Chem. 94, 5483 (1990)

14. C. Angeli, R. Cimiraglia, J.P. Malrieu, J. Chem. Phys. 117, 9138 (2002)

15. T.N. Lan, Y. Kurashige, T. Yanai, J. Chem. Theory Comput. 10, 1953 (2014)

16. B. Fernandez, P. Jørgensen, J. Byberg, J. Olsen, T. Helgaker, H.J.A. Jensen, J. Chem. Phys. 97(5), 3412 (1992)

17. P.A. Malmqvist, B.O. Roos, Chem. Phys. Lett. 155, 189 (1989)

18. M.S. Vad, P.M. N., A. Nørager, H.J.A. Jensen, J. Chem. Phys. 138, 214106 (2013)

19. W. Kutzelnigg, Z. Phys. D 15, 27 (1990)

20. J.P. Perdew, A. Ruzsinszky, L.A. Constantin, J. Sun, G.I. Csonka, J. Chem. Theory Comput. 5(4), $902(2009)$ 
21. L.A. Eriksson, in Encyclopedia of Computational Chemistry, ed. by P. von Ragué Schleyer (Wiley, Chichester, UK, 1998), pp. 952-958

22. S. Patchkovskii, G. Schreckenbach, in Calculation of NMR and EPR Parameters. Theory and Applications, ed. by M. Kaupp, M. Bühl, V.G. Malkin (Wiley-VCH, Weinheim, 2004), pp. 505-532

23. M. Kaupp, F.H. Köhler, Coord. Chem. Rev. 253(19-20), 2376 (2009)

24. F. Neese, Coord. Chem. Rev. 253(5-6), 526 (2009)

25. T. Helgaker, S. Coriani, P. Jørgensen, K. Kristensen, J. Olsen, K. Ruud, Chem. Rev. 112, 543 (2012)

26. J. Autschbach, T. Ziegler, Coord. Chem. Rev. 238/239, 83 (2003)

27. J. Autschbach, in Relativistic Methods for Chemists, Challenges and Advances in Computational Chemistry and Physics, vol. 10, ed. by M. Barysz, Y. Ishikawa (Springer, Dordrecht, 2010), chap. 12, pp. 521-598. URL http://dx.doi.org/10.1007/ 978-1-4020-9975-5_12

28. E. van Lenthe, P.E.S. Wormer, A. van der Avoird, J. Chem. Phys. 107, 2488 (1997)

29. E. Malkin, M. Repisky, S. Komorovsky, P. Mach, O.L. Malkina, V.G. Malkin, J. Chem. Phys. 134(4), 044111 (2011)

30. P. Verma, J. Autschbach, J. Chem. Theory Comput. 9, 1932 (2013)

31. S. Schmitt, P. Jost, C. van Wüllen, J. Chem. Phys. 134(19), 194113 (2011)

32. C. van Wüllen, J. Chem. Phys. 130(19), 194109 (2009)

33. J. Autschbach, J. Chem. Phys. 136, 150902 (2012)

34. L. Visscher, K. Dyall, At. Data Nucl. Data Tables 67(2), 207 (1997)

35. W. Van den Heuvel, A. Soncini, Phys. Rev. Lett. 109, 073001 (2012)

36. C. Rodowicz, Nukleonika 58, 341 (2013)

37. R. Maurice, R. Bastardis, C. de Graaf, N. Suaud, T. Mallah, N. Guihéry, J. Chem. Theory Comput. 11, 2977 (2009)

38. M.H.L. Pryce, Phys. Rev. Lett. 3, 375 (1959)

39. J.D. Axe, H.J. Stapleton, C.D. Jefries, Phys. Rev. 121, 1630 (1961)

40. P. Rigny, P. Plurien, J. Phys. Chem. Solids 28, 2589 (1967)

41. J.C. Eisenstein, M.H.L. Pryce, J. Res. Natl. Bur. Stand. Sect. A 69A, 217 (1965)

42. L.F. Chibotaru, L. Ungur, Phys. Rev. Lett. 109, 246403 (2012)

43. L. Chibotaru, A. Ceulemans, H. Bolvin, Phys. Rev. Lett. 101, 033003 (2008)

44. F.P. Notter, H. Bolvin, J. Chem. Phys. 130, 184310 (2009)

45. F. Gendron, D. Paez Hernandez, F.P. Notter, B. Pritchard, H. Bolvin, J. Autschbach, Chem. Eur. J. 20, 7994 (2014)

46. F. Gendron, B. Pritchard, H. Bolvin, J. Autschbach, Inorg. Chem. 53, 8577 (2014)

47. G. Rogez, J.N. Rebilly, A.L. Barra, L. Sorace, G. Blondin, N. Kirchner, M. Duran, J. van Slageren, S. Parsons, L. Ricard, A. Marvilliers, T. Mallah, Angew. Chem. 44, 1876 (2005)

48. D. Nelson, L.W. ter Haar, Inorg. Chem. 32, 182 (1993)

49. D. Páez Hernàndez, H. Bolvin, J. Electron. Spectrosc. Relat. Phenom. 194, 74 (2014)

50. H. Andres, E.L. Bominaar, J.M. Smith, N.A. Eckert, P.L. Holland, E. Mnck, J. Am. Chem. Soc. 124, 3012 (2002)

51. J. Autschbach, J. Phil. Trans. A 372, 20120489 (2014)

52. K. Sharkas, J. Autschbach, J. Chem. Theory Comput. p. in press. http://dx. doi.org/ $10.1021 /$ ct $500988 \mathrm{~h}$

53. L. Chibotaru, L. Ungur, J. Chem. Phys. 137, 064112 (2012) 